Draft VERSion August 30, 2021

Preprint typeset using LTEX style emulateapj v. 5/2/11

\title{
THE BLACK HOLE IN THE COMPACT, HIGH-DISPERSION GALAXY NGC 1271
}

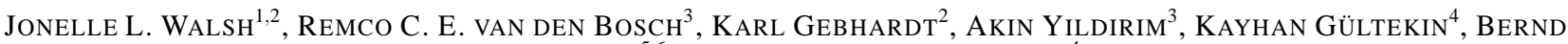 \\ HUSEMANN $^{5,6}$, AND DOUGLAS O. RICHSTONE ${ }^{4}$ \\ ${ }^{1}$ George P. and Cynthia Woods Mitchell Institute for Fundamental Physics and Astronomy, and Department of Physics and Astronomy, Texas A\&M \\ University, College Station, TX 77843, USA; walsh@physics.tamu.edu \\ ${ }^{2}$ Department of Astronomy, The University of Texas at Austin, 2515 Speedway, Stop C1400, Austin, TX 78712, USA \\ ${ }^{3}$ Max-Planck Institut für Astronomie, Königstuhl 17, D-69117 Heidelberg, Germany \\ ${ }^{4}$ Department of Astronomy, University of Michigan, 1085 S. University Ave., Ann Arbor, MI 48109, USA \\ ${ }^{5}$ European Southern Observatory, Karl-Schwarzschild-Str. 2, 85748 Garching, Germany \\ ${ }^{6}$ Leibniz Institute for Astrophysics Potsdam, An der Sternwarte 16, 14482 Potsdam, Germany \\ Draft version August 30, 2021
}

\begin{abstract}
Located in the Perseus cluster, NGC 1271 is an early-type galaxy with a small effective radius of $2.2 \mathrm{kpc}$ and a large bulge stellar velocity dispersion of $276 \mathrm{~km} \mathrm{~s}^{-1}$ for its $K$-band luminosity of $8.9 \times 10^{10} L_{\odot}$. We present a mass measurement for the black hole in this compact, high-dispersion galaxy using observations from the integral field spectrograph NIFS on the Gemini North telescope assisted by laser guide star adaptive optics, large-scale integral field unit observations with PPAK at the Calar Alto Observatory, and Hubble Space Telescope WFC3 imaging observations. We are able to map out the stellar kinematics both on small spatial scales, within the black hole sphere of influence, and on large scales that extend out to four times the galaxy's effective radius. We find that the galaxy is rapidly rotating and exhibits a sharp rise in the velocity dispersion. Through the use of orbit-based stellar dynamical models, we determine that the black hole has a mass of $\left(3.0_{-1.1}^{+1.0}\right) \times 10^{9} M_{\odot}$ and the $H$-band stellar mass-to-light ratio is $1.40_{-0.11}^{+0.13} \Upsilon_{\odot}(1 \sigma$ uncertainties $)$. NGC 1271 occupies the sparsely-populated upper end of the black hole mass distribution, but is very different from the Brightest Cluster Galaxies (BCGs) and giant elliptical galaxies that are expected to host the most massive black holes. Interestingly, the black hole mass is an order of magnitude larger than expectations based on the galaxy's bulge luminosity, but is consistent with the mass predicted using the galaxy's bulge stellar velocity dispersion. More compact, high-dispersion galaxies need to be studied using high spatial resolution observations to securely determine black hole masses, as there could be systematic differences in the black hole scaling relations between these types of galaxies and the BCGs/giant ellipticals, thereby implying different pathways for black hole and galaxy growth.
\end{abstract}

Subject headings: galaxies: elliptical and lenticular, cD - galaxies: individual (NGC 1271) - galaxies: kinematics and dynamics - galaxies: nuclei - black hole physics

\section{INTRODUCTION}

Over the past 15 years, it has become increasingly clear that supermassive black holes are an essential component of a galaxy, as demonstrated by the correlations connecting black hole masses and galaxy bulge properties (e.g., Kormendy \& Richstone 1995; Ferrarese \& Merritt 2000; Gebhardt et al. 2000; Marconi \& Hunt 2003; Gültekin et al. 2009; Kormendy \& Ho 2013). Supermassive black holes are thought to regulate galaxy properties and influence star formation via feedback processes (Silk \& Rees 1998; Fabian 1999), however the black hole - bulge relations can also arise because of the inherent averaging associated with random galaxy mergers, without the need for the black hole to actively influence its host galaxy (Peng 2007; Jahnke \& Macciò 2011). Roughly 80 dynamical black hole mass $\left(M_{\mathrm{BH}}\right)$ measurements have been made to date (Kormendy \& Ho 2013), almost exclusively through the use of high angular resolution facilities such as the Hubble Space Telescope (HST) and 8-10m ground-based telescopes with the aid of adaptive optics (AO). Despite the growing number of black hole mass measurements, the local black hole mass census is highly incomplete. Gaining a more complete picture of black hole demographics and a deeper understanding the mechanisms that drive black hole/galaxy evolution requires the secure measurement of many more black holes, particularly those at the extremes of the black hole mass scale and in a wider range of galaxy types.

With the goal of finding more objects suitable for future dynamical black hole mass measurements, the HET Massive Galaxy Survey obtained long-slit spectra of $\sim 1000$ galaxies using the Hobby-Eberly Telescope (HET) at McDonald Observatory (van den Bosch et al. 2015). The survey uncovered a sample of interesting early-type galaxies with small sizes and high stellar velocity dispersions for their luminosities. More quantitatively, these galaxies have an effective radius, $R_{e}$, below $3 \mathrm{kpc}$, a central stellar velocity dispersion larger than $250 \mathrm{~km} \mathrm{~s}^{-1}$, and $K$-band luminosities $\sim(5-25) \times 10^{10} L_{\odot}$. The HET spectra hint that these compact, high-dispersion galaxies could host some of the largest black holes known $\left(M_{\mathrm{BH}}>10^{9} M_{\odot}\right)$, and that the black holes could weigh a high fraction of its host galaxy's mass. Six example objects were highlighted in van den Bosch et al. (2012), and orbit-based stellar dynamical models were calculated for one galaxy, NGC 1277. In the case of NGC 1277, van den Bosch et al. (2012) found a $1.7 \times 10^{10} M_{\odot}$ black hole that is surprisingly $59 \%$ of the galaxy's bulge mass, or $14 \%$ of the galaxy's total mass. While Yıldırım et al. (2015) infer a similar $M_{\mathrm{BH}}$ from seeing-limited, large-scale integral field unit (IFU) data, Emsellem (2013) show a smaller black hole of a few billion solar masses can also reasonably reproduce the observed HET long-slit kinematics presented in van den Bosch et al. (2012). 
Obtaining secure black hole mass measurements for the other compact, high-dispersion galaxies found through the HET Massive Galaxy Survey is important for addressing questions concerning the upper end of the black hole - host galaxy relationships. With the present sample of black hole mass measurements, the slope, intrinsic scatter, and even the shape of the correlations for high-mass black holes are not well established (e.g., McConnell \& Ma 2013). Also, the black hole mass - stellar velocity dispersion relation $\left(M_{\mathrm{BH}}-\right.$ $\left.\sigma_{\star}\right)$ and the black hole mass - bulge luminosity relation $\left(M_{\mathrm{BH}}\right.$ $\left.-L_{\text {bul }}\right)$ are in direct conflict at the upper end, and make drastically different predictions for the inferred number density of the most massive black holes (Lauer et al. 2007). Not only are the compact, high-dispersion galaxies from the HET survey useful for filling in the poorly sampled high-mass end of the black hole relations, but also the mass estimates from $M_{\mathrm{BH}}$ $-\sigma_{\star}$ and $M_{\mathrm{BH}}-L_{\text {bul }}$ differ by a factor of at least three. Consequently, the galaxies are also useful for testing which of the correlations is more fundamental and a better predictor of $M_{\mathrm{BH}}$ at the high-mass end of the scaling relations.

While recent progress has been made in searching for, and revising measurements for, black holes with masses larger than $10^{9} M_{\odot}$ (Shen \& Gebhardt 2010; Gebhardt et al. 2011; McConnell et al. 2011, 2012; van den Bosch et al. 2012; Walsh et al. 2010, 2013; Rusli et al. 2013), many of these galaxies are giant ellipticals or Brightest Cluster Galaxies (BCGs), which are often large (with $R_{e}>10 \mathrm{kpc}$; e.g., Dalla Bontà et al. 2009), have cored surface brightness profiles, and are dispersion-supported showing little to no rotation. In contrast, the compact, high-dispersion galaxies found through the HET survey are small, rapidly rotating, and generally exhibit cuspy surface brightness profiles. Such host galaxy environments haven't been extensively explored on the black hole host galaxy relationships. Besides NGC 1277, only the compact galaxies NGC 1332 (Rusli et al. 2011), NGC 4342 (Cretton \& van den Bosch 1999), NGC 4486B (Kormendy et al. 1997), and M60-UCD1 (Seth et al. 2014) have dynamical black hole mass measurements, with NGC 1332 and NGC 4342 being most like NGC 1277 . For these galaxies, the black hole mass measurements are in agreement with $M_{\mathrm{BH}}$ $-\sigma_{\star}$ given the intrinsic scatter of the relation, but are positive outliers from the $M_{\mathrm{BH}}-L_{\mathrm{bul}}$ relation (Kormendy \& Ho 2013). We note that there are uncertainties associated with the bulge luminosity for NGC 1332 (see Kormendy \& Ho 2013 for details) and the black hole mass measurement for NGC $4486 \mathrm{~B}$ (see Gültekin et al. 2009 for details). Also, tidal stripping is believed to be the cause of the over-massive black hole in the ultracompact dwarf galaxy M60-UCD1 (Seth et al. 2014), and there is some debate as to whether NGC 4486B and NGC 4342 have been stripped as well (e.g., Faber 1973; Bogdan et al. 2012; Blom et al. 2014). Nevertheless, additional similar galaxies need to be studied because there could be systematic differences in the scaling relations between the compact, high-dispersion galaxies and the giant ellipticals/BCGs. If so, that would imply that the black holes in the two types of galaxies grew in different ways.

While the compact, high-dispersion galaxies are unusual in the present-day Universe, they are qualitatively similar to the typical $z \sim 2$ quiescent galaxies, which are also small, have disk-like features, and could have high velocity dispersions (Zirm et al. 2007; van Dokkum et al. 2009; van der Wel et al. 2011). The $z \sim 2$ red nuggets are believed to be the progenitors of the massive early-type galaxies seen today, evolved in size and mass (e.g., van Dokkum et al. 2010). Thus, the compact, high-dispersion galaxies found through the HET survey could provide clues to the link between local galaxies and the $z \sim 2$ red nuggets, the ultra compact sub-mm galaxies at $z \sim 3$ (Toft et al. 2014), and the early massive black holes found in $z>6$ quasars (Fabian et al. 2013).

We have begun to obtain the imaging and spectroscopic observations necessary for a detailed examination of the compact, high-dispersion galaxies from the HET Massive Galaxy Survey. This includes HST and AO-assisted IFU observations to probe the region over which the black hole dominates the galaxy's potential (the black hole sphere of influence; $\left.r_{\text {sphere }}=G M_{\mathrm{BH}} / \sigma_{\star}^{2}\right)$, and IFU observations that sample the large-scale stellar kinematics out to several effective radii. In this paper, we focus on measuring the mass of the black hole for the first compact, high-dispersion galaxy for which we have completed AO IFU observations. NGC 1271 has not been widely investigated in the literature and is given an uncertain SB0 classification according to the NASA/IPAC Extragalactic Database (NED). The galaxy is located within the Perseus cluster, at $z=0.0192$, and we adopt a distance to NGC 1271 of $80 \mathrm{Mpc}$, which is the Hubble flow distance derived from the Mould et al. (2000) Virgo + Great Attractor + Shapley Supercluster Infall velocity field model assuming a Hubble constant of $H_{0}=70.5 \mathrm{~km} \mathrm{~s}^{-1} \mathrm{Mpc}^{-1}$, a matter density of $\Omega_{M}=0.27$ and a cosmological constant of $\Omega_{\Lambda}=0.73$. The Sloan Digital Sky Survey $g-i$ color is 1.6 and absolute $r$-band magnitude is -20.8 for the galaxy. Long-slit spectra of NGC 1271 were obtained through the HET Massive Galaxy Survey, and the reported $[\mathrm{N} \mathrm{II}] / \mathrm{H} \alpha$ and $[\mathrm{O}$ III $] / \mathrm{H} \beta$ emission-line ratios measured within a 3." 5 aperture (van den Bosch et al. 2015) place NGC 1271 just within the composite galaxies section near the active galactic nuclei side of Kewley et al. (2006). Also, van den Bosch et al. (2015) do not find the presence of any broad emission lines.

In Section 2, we describe the imaging and spectroscopic observations, including the data reduction procedures. We present the luminous mass model for the galaxy in Section 3 and the stellar kinematics in Section 4. Our determination of the point-spread function (PSF) for the spectroscopic observations is discussed in Section 5, and an overview of the orbit-based stellar dynamical models is given in Section 6. In Section 7, we present the results of the models and examine possible sources of systematic uncertainty. Finally, in Sections 8 and 9, we study the galaxy's orbital structure, discuss NGC 1271 within the context of the $M_{\mathrm{BH}}$ - host galaxy relationships, and summarize our findings.

\section{OBSERVATIONS}

For NGC 1271, we obtained imaging observations with the HST Wide-Field Camera 3 (WFC3) in order to measure the galaxy's surface brightness distribution. We also acquired spectra with the Near-infrared Integral Field Spectrometer (NIFS; McGregor et al. 2003) on the $8.1 \mathrm{~m}$ Gemini North telescope assisted by the ALTtitude conjugate Adaptive optics for the InfraRed (Herriot et al. 2000; Boccas et al. 2006) system. The NIFS data is important for constraining $M_{\mathrm{BH}}$, as it resolves the black hole sphere of influence. We also acquired large-scale spectra with the Postdam Multi Aperture Spectrograph (PMAS; Roth et al. 2005) in the Pmas fiber PAcK (PPAK; Verheijen et al. 2004; Kelz et al. 2006) mode at the $3.5 \mathrm{~m}$ telescope at Calar Alto Observatory. Although long-slit spectroscopic observations along the galaxy major axis have been previously made using the HET, measuring a large-scale, 
two-dimensional (2D) velocity field is preferable over a single slit observation for constraining the stellar mass-to-light ratio and the stellar orbital distribution. Hence, we use the PPAK IFU observations in place of the major-axis HET measurements. Below we describe the WFC3, NIFS, and PPAK observations and data reduction methods.

\subsection{HST Imaging}

We observed NGC 1271 with HST WFC3 and the IR/F160W filter under program GO-13050. The observation was composed of three dithered full array exposures of 450 $\mathrm{s}$, and four dithered subarray exposures of $1.7 \mathrm{~s}$, leading to a total integration time of $1354 \mathrm{~s}$. The short subarray exposures were chosen to ensure the nucleus would not become saturated. The flattened, calibrated images were corrected for geometric distortions, cleaned, and combined using AstroDrizzle (Gonzaga et al. 2012). Since the exposures were dominated by galaxy light, we found that the standard AstroDrizzle sky subtraction overestimated the background flux. We therefore manually measured the background level in each of the images. For the full array images, we measured the flux from the corners of each image, while for the subarray exposures we measured the flux difference between the sky-subtracted full frames and the subarray frames. With the background level determined, the exposures were combined to produce a supersampled image with a spatial resolution of 0.106 pixel $^{-1}$. Not only is the HST image suitable for determining the luminous distribution on near the black hole, but due to the small size of NGC 1271, we were also able to measure the luminosity out to larger galaxy scales, extending to $\sim 5 R_{e}$ (adopting an effective radius of 5 .' 6 , or $2.2 \mathrm{kpc}$, measured from a single component Sérsic fit to the HST F160W image; see Section 8.2).

\subsection{NIFS Spectroscopy}

The NIFS laser guide star (LGS) AO observations were acquired over three nights, on 2012 Dec 27, 2012 Dec 29, and 2013 Jan 8, in queue mode under program GN-2012B-Q-51. We used the $H+K$ filter and the $K$ grating with a central wavelength of $2.2 \mu \mathrm{m}$ to obtain spectra over a $3^{\prime \prime} \times 3^{\prime \prime}$ field-of-view and a spectral resolution of $\mathrm{R} \sim 5290$. We recorded $900 \mathrm{~s}$ exposures of the galaxy nucleus, following an Object-Sky-Object observing sequence, totaling 3 hours of on-source integration. An $R=17.8$ mag star located $17^{\prime \prime}$ away from the galaxy was used as the tip-tilt reference. In addition, we observed the tiptilt star to monitor the PSF during each of the three nights and the A0 V stars HIP 10559 and HIP 22842 for telluric correction.

The data were reduced using $\operatorname{IRAF}^{7}$ tasks within the Gemini/NIFS package version 1.11, utilizing the example NIFS processing scripts ${ }^{8}$. The reduction included sky subtraction, flat fielding, interpolation over bad pixels, cosmic-ray cleaning, and spatial rectification and wavelength calibration using Ronchi mask and arc lamp exposures. The spectra were then corrected for telluric features, using an A0 V star spectrum, after interpolating over the $\mathrm{Br} \gamma$ absorption line and dividing by a black body with a temperature of $9480 \mathrm{~K}$. Next, a data cube was produced, having $x$ and $y$ spatial dimensions, each

\footnotetext{
7 IRAF is distributed by the National Optical Astronomy Observatory, which is operated by the Association of Universities for Research in Astronomy under cooperative agreement with the National Science Foundation

8 http://www.gemini.edu/sciops/instruments/nifs/data-format-and-
}

with a scale of 0. . $^{\prime} 05$ pixel $^{-1}$, and one spectral dimension, $\lambda$, using a common wavelength range and sampling for the individual science exposures. The relative spatial positions between the data cubes were determined by summing along the wavelength axis and cross-correlating the resulting flux maps. The offsets were used to align and combine the 12 individual exposures, generating the final data cube of the galaxy. Data reduction of the PSF star observations followed a similar procedure.

\subsection{PPAK Spectroscopy}

The PPAK observations of NGC 1271 were acquired as part of a campaign to obtain large-scale spectroscopy of the compact, high-dispersion galaxies. The observations, data reduction, and kinematic measurements for NGC 1271 will be presented in Yildirım et al. (in prep), but follow closely the PPAK observations for two other compact, high-dispersion galaxies described in Yildırım et al. (2015). For completeness, we briefly review the pertinent information below and in Section 4.2.

The wide-field IFU observations were taken over three nights, from 2013 Jan 4-6, using the V500 grating to provide coverage of $4200-7000 \AA$ with a spectral resolution of $\mathrm{R} \sim 850$ at $5000 \AA$. We used three dithers to fully sample the 3312 ."7-diameter fibers, to increase the spatial resolution of the data, and to address effects of vignetting and bad pixels. An additional 36 fibers, located $72^{\prime \prime}$ away from the center of the instrument field-of-view, were used to measure the sky. During each of the three nights, two 1200 s science exposures were taken at each of the three dither positions, leading to a total of 6 hours of on-source integration. The data reduction followed the procedure adopted for the Calar Alto Legacy Integral Field Spectroscopy Area Survey. The main steps included bias subtraction, flat-fielding, cosmic ray cleaning, extraction of spectra, wavelength calibration, sky subtraction, and flux calibration using spectrophotometric standard stars. Spectra from the three pointings were then combined and resampled into a data cube, followed by a correction for differential atmospheric refraction. We note that the line spread function is measured as a function of wavelength and fiber position from arc lamps during the wavelength calibration step, and then homogenized to a common value prior to the extraction of the PPAK kinematics. The details of these steps are discussed at length by Sánchez et al. (2012) and Husemann et al. (2013) and we refer the reader to those publications for additional information.

\section{CONSTRUCTING THE LUMINOUS MASS MODEL}

We generated a luminous mass model for NGC 1271 by parameterizing the HST WFC3 F160W image as the sum of 2D Gaussians using the Multi-Gaussian Expansion (MGE) formalism (Monnet et al. 1992; Emsellem et al. 1994). The MGE method is able to reproduce a wide range of galaxy surface brightness profiles and allows for an analytic deprojection to determine the intrinsic luminosity density. Here, we use the image decomposition package Galfit (Peng et al. 2010) because it takes into consideration an error map during the fit and allows for the detailed examination of model residuals, but we utilize the implementation of Cappellari (2002) to determine suitable starting parameter values for the initial run with Galfit. When constructing the MGE model, we account for the WFC3 PSF, which we adopt from van der Wel et al. (2012). This PSF was generated with Tiny Tim (Krist \& Hook 

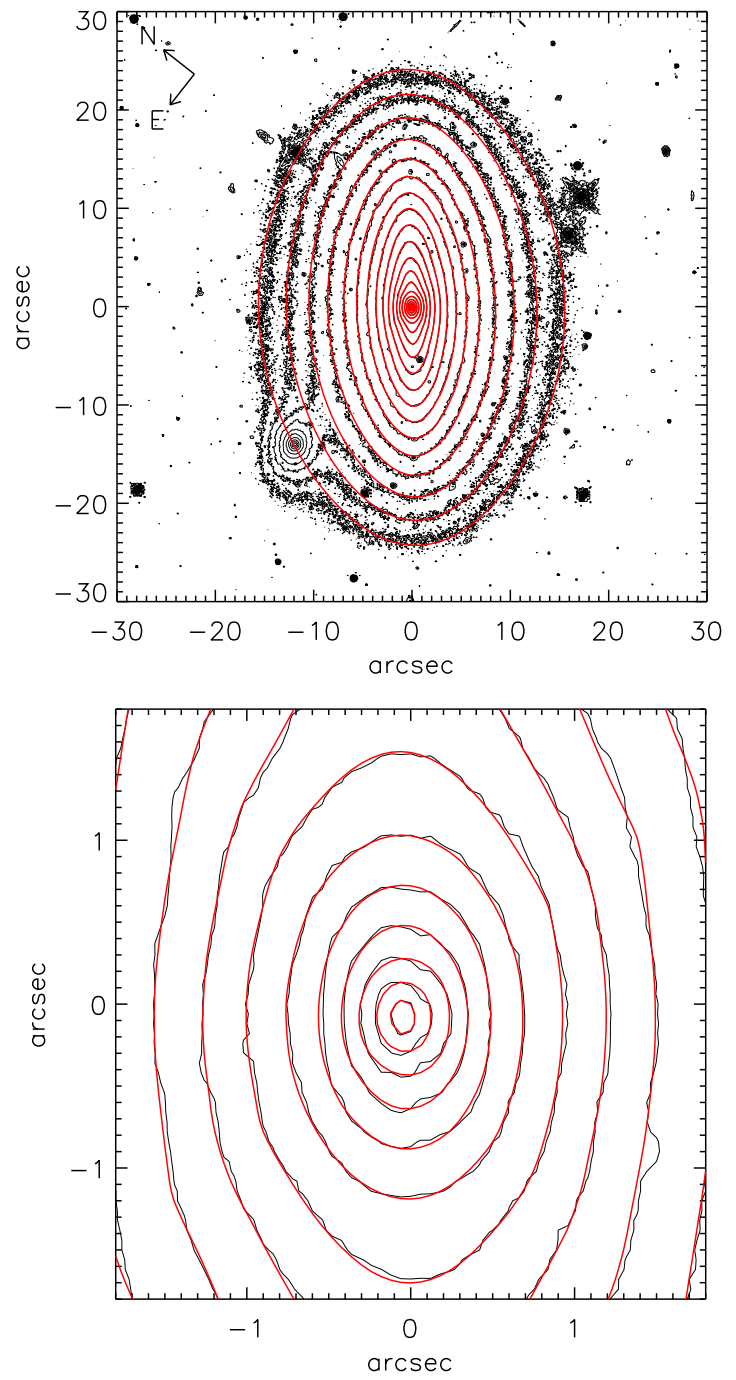

FIG. 1.- Isophotes of the MGE model (red) are compared to the HST WFC3 F160W image (top) and to the inner 3 !' $^{\prime} \times 33^{\prime \prime} 6$ region of the image (bottom). Contours are logarithmically spaced, but arbitrary. Foreground stars and galaxies were masked during the MGE fit.

2004) for the F160W filter at the center of the WFC3 detector assuming a $\mathrm{G} 2 \mathrm{~V}$ spectral type, and drizzled to produce a PSF with the same spatial scale as our final science image of NGC 1271. In addition, we identified foreground objects using the program SExtractor (Bertin \& Arnouts 1996) and masked these regions during the MGE fit.

The final MGE model contained 11 components, where each Gaussian was set to have the same position angle and center and the projected axis ratio $\left(q^{\prime}\right)$ was required to be larger than 0.25 . Due to the degeneracy associated with fitting a large number of Gaussians, we chose to restrict $q^{\prime}>0.25$ in order to avoid highly flattened components that place very stringent constraints on the viewing angles for which a model can be deprojected. The final MGE model is an good representation of the galaxy, as can be seen in Figure 1. We present the final MGE parameters, after correction for galactic extinction using the Schlafly \& Finkbeiner (2011) value given by NED, in Table 1.

\section{EXTRACTING THE STELLAR KINEMATICS}

TABLE 1

MGE PARAMETERS

\begin{tabular}{lccc}
\hline \hline$j$ & $\log I_{j}\left(\mathrm{~L}_{\odot, H} \mathrm{pc}^{-2}\right)$ & $\sigma_{j}^{\prime}\left({ }^{\prime \prime}\right)$ & $q_{j}^{\prime}$ \\
$(1)$ & $(2)$ & $(3)$ & $(4)$ \\
\hline 1 & 6.074 & 0.073 & 0.25 \\
2 & 4.778 & 0.175 & 0.83 \\
3 & 4.786 & 0.399 & 0.56 \\
4 & 4.183 & 0.765 & 0.77 \\
5 & 3.172 & 1.838 & 0.66 \\
6 & 3.756 & 2.018 & 0.26 \\
7 & 3.391 & 4.306 & 0.25 \\
8 & 3.054 & 5.857 & 0.38 \\
9 & 2.474 & 8.876 & 0.49 \\
10 & 1.879 & 12.946 & 0.72 \\
11 & 0.889 & 24.772 & 0.99 \\
\hline
\end{tabular}

NOTE. - The component number is listed in column (1), the central surface brightness, using a galactic extinction of 0.085 and a solar absolute magnitude of 3.33 , is provided in column (2), the dispersion along the major axis is given in column (3), and the axis ratio is presented in column (4). The components all have of a position angle of $-50.3^{\circ}$ and projected quantities are denoted with primed variables.

From the NIFS and PPAK data cubes, we measured the line-of-sight velocity distribution (LOSVD) as a function of spatial location. The LOSVD was described using the first four Gauss-Hermite $(\mathrm{GH})$ moments: the radial velocity $(V)$, the velocity dispersion $(\sigma)$, and $h_{3}$ and $h_{4}$, which describe the LOSVD's asymmetric and symmetric deviations from a Gaussian. High signal-to-noise (S/N) spectra, typically $\gtrsim 30$, are required in order to reliably extract the higher order $\mathrm{GH}$ moments (e.g., van der Marel \& Franx 1993; Bender et al. 1994), and thus we use the Voronoi binning algorithm (Cappellari \& Copin 2003) in order to construct spatial bins that optimize the balance between spatial resolution and S/N. We then used the penalized pixel fitting (pPXF) method of Cappellari \& Emsellem (2004) to measure the stellar kinematics in each bin. This procedure determines the best-fitting LOSVD by convolving with a stellar template to match the observed galaxy spectra. Errors on the kinematics were determined using Monte Carlo simulations, in which random Gaussian noise was added to the spectrum based upon the pPXF model residuals. We performed 100 realizations and from the distributions measured the standard deviation to determine $1 \sigma$ uncertainties. During the Monte Carlo runs, the penalization term was set to zero to produce realistic errors.

\subsection{NIFS Kinematics}

We measured the stellar kinematics from the three primary $K$-band $\mathrm{CO}$ bandheads $\left[(2-0)^{12} \mathrm{CO},(2-1)^{12} \mathrm{CO}\right.$, and $(4-2)^{12} \mathrm{CO}$ ] in 127 spatial bins by using pPXF to fit the wavelength region between $2.26-2.42 \mu \mathrm{m}$. We made use of the NIFS Spectral Template Library v2.09 (Winge et al. 2009), which contains 28 stars observed using the NIFS IFU with the $K$ grating and $H+K$ filter. The library includes spectral types ranging from G8 - M5 giant stars, K3 - M3 supergiants, and a G8 II star.

We first created an optimal stellar template by fitting a high $\mathrm{S} / \mathrm{N}$ spectrum, constructed by adding together all spectra in the galaxy data cube. This optimal template was composed of six stars, and was dominated by M5 III, M3 III, and K3 Iab stars that make up $37 \%, 26 \%$, and $21 \%$ of the total flux, respectively. Next, we measured the $\mathrm{GH}$ moments in each

9 http://www.gemini.edu/sciops/instruments/nearir-resources/spectraltemplates 
spatial bin with pPXF by keeping the relative weights of the stars that make up the template fixed, but allowing the coefficients of a second degree additive Legendre polynomial and a second degree multiplicative Legendre polynomial to vary. Such polynomials are needed to account for differences in the optimal stellar template and the galaxy spectra shape, as the continuum of the stars in the NIFS Spectral Template Library has been previously removed. The kinematics were in good agreement with those measured when fitting a new optimal stellar template to each spatial bin and fitting only two GH moments. Finally, we bi-symmetrize the kinematics using the machinery presented van den Bosch \& de Zeeuw (2010). Since the dynamical models are only able to produce symmetric kinematics, this step is commonly performed (e.g., Gebhardt et al. 2003; Cappellari et al. 2006; Onken et al. 2014 ) in order to reduce the noise in the observations. With the symmetrization routine, the systematic offsets in the odd GH moments, such as the galaxy's recession velocity, are removed as well.

From the NIFS data, we find that the galaxy is rotating quickly, with stars reaching velocities of $\pm 226 \mathrm{~km} \mathrm{~s}^{-1}$. There is also a sharp peak in the velocity dispersion, which rises from $205 \mathrm{~km} \mathrm{~s}^{-1}$ at a radius of $\sim 1^{\prime \prime}$ to $396 \mathrm{~km} \mathrm{~s}^{-1}$ at the center. The map of $h_{3}$ is anti-correlated with the velocity map, while $h_{4}$ shows a slight increase at the center. The $\mathrm{S} / \mathrm{N}$ in each spatial bin (measured as the ratio between the median value of the spectrum and standard deviation of the pPXF model residuals) ranged between 33 and 96 with a median value of 66. Therefore, we were able to place excellent constraints on the kinematics, with median errors over all spatial bins of $7 \mathrm{~km}$ $\mathrm{s}^{-1}, 9 \mathrm{~km} \mathrm{~s}^{-1}, 0.02$, and 0.02 for $V, \sigma, h_{3}$, and $h_{4}$, respectively. We present example spectra and fits with pPXF at three different locations within the NIFS data cube in Figure 2 and the bi-symmetrized NIFS kinematics in Table 2.

\subsection{PPAK Kinematics}

We measured the stellar kinematics in 268 spatial bins over a wavelength range of 4200-7000 $\AA$, which includes a number of absorption features such as the $\mathrm{H} \beta, \mathrm{Mg} I b$, and $\mathrm{Fe}$ 5015 lines. During the fit with pPXF, we masked sky features and emission lines and included a 15th degree additive polynomial. The kinematics were extracted using the Indo-U.S. Library of Coudé Feed Stellar Spectra (Valdes et al. 2004), and the optimal stellar template was dominated by G9 V, G9 III, K0 III, and A0p stars. As a final step, we bi-symmetrize the kinematics and subtract off the systematic offsets in the odd GH moments using the procedure in van den Bosch \& de Zeeuw (2010).

The kinematics from the PPAK data were measured out to $\sim 24^{\prime \prime}$, or $\sim 4 R_{e}$. The large-scale kinematics exhibit features that are similar to the measurements made from the high spatial resolution NIFS data. In particular, the stars show rotation with velocities of \pm 231 , a peak in the velocity dispersion to values of $297 \mathrm{~km} \mathrm{~s}^{-1}$ from $102 \mathrm{~km} \mathrm{~s}^{-1}$ at radius of $\sim 24^{\prime \prime}$, and there is a $h_{3}-V$ anti-correlation. The difference in the peak velocity dispersions measured from the PPAK and NIFS data can be attributed to the very different spatial resolutions of the two data sets. Typical errors on the PPAK kinematics for $V, \sigma$, $h_{3}$, and $h_{4}$ are $8 \mathrm{~km} \mathrm{~s}^{-1}, 12 \mathrm{~km} \mathrm{~s}^{-1}, 0.04$, and 0.05 . We present example fits to the galaxy spectra at several spatial locations in Figure 3 and provide the bi-symmetrized PPAK kinematics in Table 3.

NGC 1271 has an uncertain SB0 classification according to

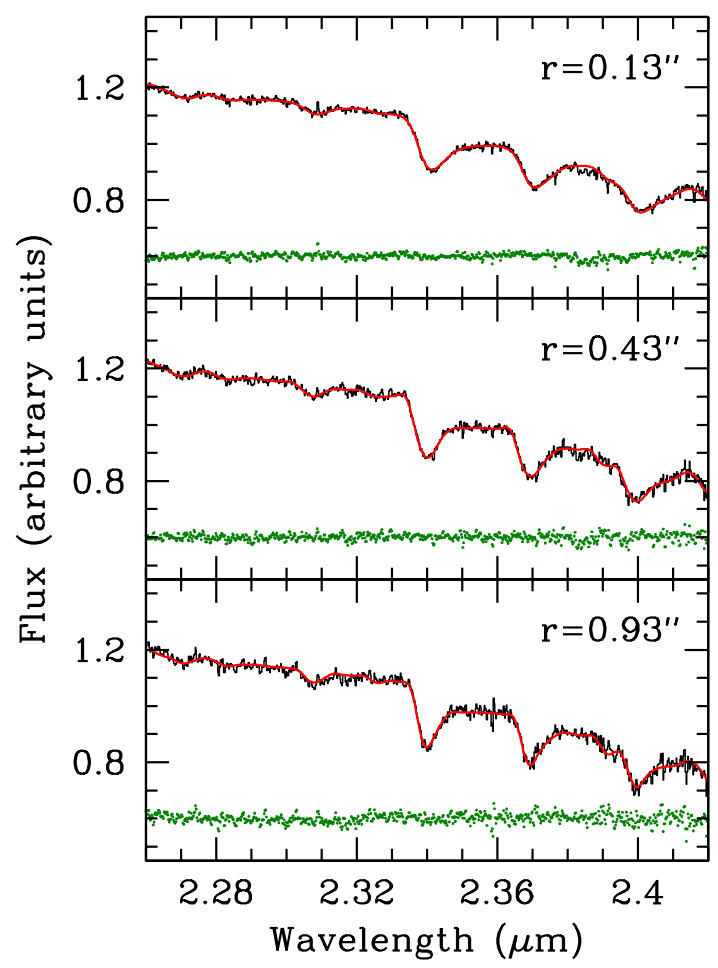

FIG. 2.- Shown in the top, middle, and bottom panels, respectively, are example NIFS spectra extracted from three spatial locations: a single spaxel located near the nucleus, a bin containing five spaxels at an intermediate distance from the galaxy center, and one of the outermost bins composed of 28 spaxels. Overplotted in red is the optimal stellar template convolved with the best-fitting LOSVD. The model residuals are shown in green, and have been shifted by an arbitrary amount.

NED, but we do not see an obvious bar feature in the HST F160W image or in the PPAK/NIFS kinematics. N-body simulations have shown that common kinematic signatures associated with bars include a "double-hump" feature in the rotation curve and an $h_{3}-V$ correlation over the projected length of the bar (Bureau \& Athanassoula 2005). Instead, even the unsymmetrized kinematics clearly show a smooth increase in the radial velocity from the southeast side of the galaxy to the northwest side, and that $h_{3}$ is anti-correlated with $V$, as is expected for axisymmetric systems.

\section{MEASURING THE PSF}

The PSF of the NIFS and PPAK observations are important inputs into the stellar dynamical models. In order to estimate these quantities, we convolve the MGE model presented in Section 3 with the sum of two concentric, circular 2D Gaussians in order to match the collapsed NIFS and PPAK data cubes. The PSF is parameterized by the dispersion and relative weight of each Gaussian component. We find dispersions of $0 . " 16$ and $0 . " 43$ with relative weights of 0.61 and 0.39 , respectively, for the NIFS PSF, while the PPAK PSF can be described with dispersions of 1. "52 and 5."45 with relative weights of 0.82 and 0.18 . In addition, the comparison between the HST image and the collapsed data cubes allows for the center of the NIFS and PPAK apertures to be defined. The core of the NIFS PSF is larger than expected for AO observations (e.g., Krajnović et al. 2009; Seth et al. 2014), which is likely the result of using a fairly faint, off-axis tip-tilt star. In Section 7.1, we test the effect of our assumed NIFS PSF on the inferred black hole mass by instead estimating the PSF 
TABLE 2

NIFS KINEMATICS

\begin{tabular}{cccccccccc}
\hline \hline$x\left({ }^{\prime \prime}\right)$ & $y\left({ }^{\prime \prime}\right)$ & $V\left(\mathrm{~km} \mathrm{~s}^{-1}\right)$ & $\Delta V\left(\mathrm{~km} \mathrm{~s}^{-1}\right)$ & $\sigma\left(\mathrm{km} \mathrm{s}^{-1}\right)$ & $\Delta \sigma\left(\mathrm{km} \mathrm{s}^{-1}\right)$ & $h_{3}$ & $\Delta h_{3}$ & $h_{4}$ & $\Delta h_{4}$ \\
$(1)$ & $(2)$ & $(3)$ & $(4)$ & $(5)$ & $(6)$ & $(7)$ & $(8)$ & $(9)$ & $(10)$ \\
\hline-0.027 & 0.022 & -8.879 & 7.817 & 396.368 & 10.793 & 0.017 & 0.016 & 0.029 & 0.017 \\
-0.027 & -0.028 & -38.385 & 6.554 & 394.762 & 8.844 & 0.017 & 0.014 & 0.033 & 0.018 \\
0.055 & 0.022 & 37.125 & 5.608 & 395.394 & 7.818 & -0.015 & 0.012 & 0.031 & 0.015 \\
0.055 & -0.028 & 11.045 & 6.073 & 392.972 & 8.368 & -0.001 & 0.012 & 0.029 & 0.014 \\
-0.077 & -0.003 & -45.778 & 6.763 & 392.601 & 9.207 & 0.026 & 0.014 & 0.028 & 0.017
\end{tabular}

NOTE - Table 2 is published in its entirety in the electronic edition of ApJ. A portion is shown here for guidance regarding its form and content. Columns (1) and (2) are the $x$ and $y$ Voronoi bin generators, measured relative to the galaxy center. Columns (3) - (8) provide the bi-symmetrized NIFS kinematics and errors. The position angle is $141.14^{\circ}$, measured counter-clockwise from the galaxy's major axis to $x$.

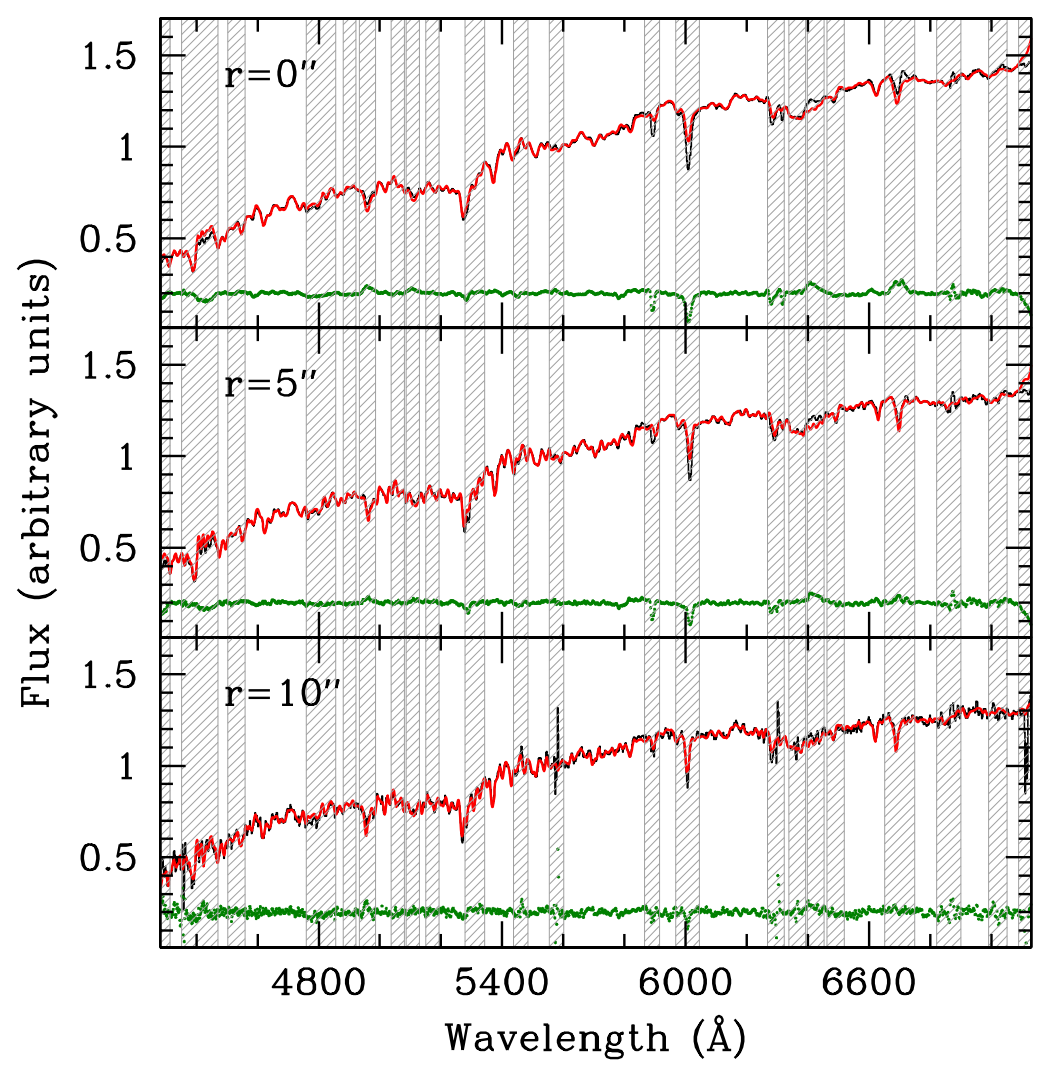

FIG. 3.- Shown in the top, middle, and bottom panels, respectively, are example PPAK spectra extracted from three spatial locations. Overplotted in red is the optimal stellar template convolved with the best-fitting LOSVD, and the gray shaded boxes denote the wavelength regions excluded during the spectral fitting, due to the presence of emission lines or sky lines. The model residuals are shown in green, and have been shifted by an arbitrary amount.

TABLE 3

PPAK KINEMATICS

\begin{tabular}{cccccccccc}
\hline \hline$x\left(^{\prime \prime}\right)$ & $y\left(^{(\prime \prime}\right)$ & $V\left(\mathrm{~km} \mathrm{~s}^{-1}\right)$ & $\Delta V\left(\mathrm{~km} \mathrm{~s}^{-1}\right)$ & $\sigma\left(\mathrm{km} \mathrm{s}^{-1}\right)$ & $\Delta \sigma\left(\mathrm{km} \mathrm{s}^{-1}\right)$ & $h_{3}$ & $\Delta h_{3}$ & $h_{4}$ & $\Delta h_{4}$ \\
$(1)$ & $(2)$ & $(3)$ & $(4)$ & $(5)$ & $(6)$ & $(7)$ & $(8)$ & $(9)$ & $(10)$ \\
\hline-0.063 & -0.208 & -28.540 & 4.759 & 296.999 & 6.723 & 0.010 & 0.013 & 0.027 & 0.016 \\
-0.063 & 0.792 & 18.003 & 4.564 & 294.131 & 6.221 & -0.013 & 0.013 & 0.025 & 0.016 \\
0.937 & -0.208 & 60.969 & 4.491 & 282.870 & 5.858 & -0.030 & 0.013 & 0.035 & 0.017 \\
-1.063 & -0.208 & -85.825 & 4.277 & 278.884 & 5.984 & 0.043 & 0.012 & 0.036 & 0.017 \\
-0.063 & -1.208 & -60.969 & 4.491 & 282.870 & 5.858 & 0.030 & 0.013 & 0.035 & 0.017 \\
& & & & & & & & &
\end{tabular}

NOTE. - Table 3 is published in its entirety in the electronic edition of ApJ. A portion is shown here for guidance regarding its form and content. Columns (1) and (2) are the $x$ and $y$ Voronoi bin generators, measured relative to the galaxy center. Columns (3) - (8) provide the bi-symmetrized PPAK kinematics and errors. The position angle is $140.71^{\circ}$, measured counter-clockwise from the galaxy's major axis to $x$. 
from NIFS observations we acquired of the tip-tilt star itself.

\section{ORBIT-BASED MODELS}

Black hole masses are often measured from stellar kinematics by constructing dynamical models based upon the Schwarzschild superposition method (Schwarzschild 1979), and here we use the three-integral, triaxial Schwarzschild code of van den Bosch et al. (2008). This technique finds a self-consistent distribution function from the observables without any assumptions about the orbital anisotropy. In the model, the black hole, the stars, and dark matter all contribute to the galaxy's gravitational potential. The stellar potential is determined by deprojecting the observed surface brightness assuming a viewing orientation and a stellar mass-to-light ratio $(\Upsilon)$ that is constant with radius. We then generate a representative orbit library in the potential, and the orbits are numerically integrated while keeping track of their intrinsic and projected properties. During the modeling, the effects of the PSF and aperture binning are taken into account. Finally, we assign weights to each orbit such that the superposition matches the observed kinematics and total light distribution. We calculate many models varying the parameters of interest $\left(M_{\mathrm{BH}}, \Upsilon\right.$, the viewing orientation parameters, and the dark matter halo parameters), and the best-fit model is the one with the lowest $\chi^{2}$.

\subsection{Application to NGC 1271}

For NGC 1271, we adopt a (nearly) oblate axisymmetric shape, with an intermediate to long axis ratio of 0.99 . We assume axisymmetry given that NGC 1271 looks highly flattened and exhibits rapid rotation, and we do not find any evidence for kinematic twists. With this assumption, the inclination angle $(i)$ is the only viewing orientation parameter needed to describe the galaxy's intrinsic shape. In our final model, we adopt $i=83^{\circ}$ (where $i=90^{\circ}$ corresponds to an edge-on view). The MGE description of NGC 1271 presented in Section 3 includes a couple of flat Gaussian components, which can only be deprojected for angles $77 \lesssim i \lesssim 90^{\circ}$. Therefore, we chose to run models at a fixed inclination angle that lies midway between the extremes. Our choice of inclination angle is further supported by a nuclear dust disk that appears highly inclined in an HST WFC3 F814W image. While the F814W image clearly shows a regular dust lane, the F160W image and the $K$-band NIFS data cube used in our analysis do not appear to be significantly affected by dust. In addition, the NGC 1271 models include a spherically symmetric dark matter halo following a Navarro-Frenk-White (NFW) form (Navarro et al. 1996). The parameters describing the NFW halo are the concentration index $(c)$ and the fraction of dark matter $\left(f_{\mathrm{DM}}\right)$, where $f_{\mathrm{DM}} \equiv M_{\mathrm{DM}} / M_{\star}$ and $M_{\mathrm{DM}}$ is the halo virial mass and $M_{\star}$ is the stellar mass. Hence, our models have four free parameters: $M_{\mathrm{BH}}, \Upsilon_{H}, c$, and $f_{\mathrm{DM}}$.

We began by calculating models on a coarse grid that spanned a wide range of values before generating models on a smaller, more finely sampled grid focused around the minimum $\chi^{2}$. Ultimately, our final model grid contained $21 M_{\mathrm{BH}}$ values, $31 \Upsilon_{H}$ values, $8 c$ values, and $20 f_{\mathrm{DM}}$ values with $\log \left(M_{\mathrm{BH}} / M_{\odot}\right) \in[9.2,10.2], \Upsilon_{H}\left(\Upsilon_{\odot}\right) \in[0.5,2.0], c \in[2,16]$, and $f_{\mathrm{DM}} \in[50,1000]$. The orbit library samples 29 equipotential shells with radii between $0 .{ }^{\prime \prime} 003$ to $100^{\prime \prime}$, and 8 angular and 8 radial values at each energy. We note that triaxial orbit families (e.g., box orbits) continue to be included in our orbital libraries because we are running a triaxial Schwarzschild code in the axisymmetric limit. Moreover, we employ a dithering method, in which 125 orbits with adjacent initial conditions are bundled together, to establish a smooth distribution function when constructing Schwarzschild models. Thus, the galaxy models are made with 696,000 orbits. The models were fit to the observed NIFS and PPAK kinematics, where $4 \mathrm{GH}$ moments were measured in a total of 395 bins, resulting in 1580 observables.

\section{MODELING RESULTS}

The results of the final model grid are summarized in Figure 4 , which shows the $\chi^{2}$ as a function of $M_{\mathrm{BH}}, \Upsilon_{H}, c$, and $f_{\mathrm{DM}}$ after marginalizing over the other three parameters. We find best-fit values of $M_{\mathrm{BH}}=3.0 \times 10^{9} M_{\odot}, \Upsilon_{H}=1.40 \Upsilon_{\odot}$, $c=16$, and $f_{\mathrm{DM}}=50$ (corresponding to $M_{\mathrm{DM}}=5.0 \times 10^{12} M_{\odot}$ ). The comparison between the observed NIFS kinematics and model predictions is shown in Figure 5 and the comparison between the PPAK data and model predictions is displayed in Figure 6. The model is an excellent match to the observed kinematics, and is able to reproduce the sharp increase in the velocity dispersion and the slight peak in $h_{4}$ at the nucleus. The $\chi^{2}$ per degree of freedom $\left(\chi_{\nu}^{2}\right)$ is 0.3 . If the unsymmetrized kinematics are used instead, the best-fit model has $\chi_{\nu}^{2}=1.3$ and an $M_{\mathrm{BH}}$ and $\Upsilon_{H}$ that are consistent within the final uncertainties given below in Section 7.1.

As can be seen in Figure 4, we are able to place strong constraints on $M_{\mathrm{BH}}$ and $\Upsilon_{H}$. By searching for the range of $M_{\mathrm{BH}}$, or $\Upsilon_{H}$, values that caused the minimum $\chi^{2}$ to increase by 1 and 9 , we estimate the $1 \sigma$ and $3 \sigma$ model fitting uncertainties. We find $M_{\mathrm{BH}}=\left(3.0_{-0.6}^{+0.1}\right) \times 10^{9} M_{\odot}(1 \sigma)$ and $M_{\mathrm{BH}}=\left(3.0_{-1.0}^{+0.9}\right) \times 10^{9} M_{\odot}(3 \sigma)$, as well as $\Upsilon_{H}=1.40_{-0.02}^{+0.05} \Upsilon_{\odot}$ $(1 \sigma)$ and $\Upsilon_{H}=1.40_{-0.15}^{+0.16} \Upsilon_{\odot}(3 \sigma)$. In contrast, the dark halo parameters are not well constrained, and the $c$ and $f_{\mathrm{DM}}$ values are highly uncertain. Specifically, the marginalized $\chi^{2}$ curve for $c$ is unconstrained at the upper end, with $c>5$ at the $3 \sigma$ level. Although the best-fit value for $c$ is at the maximum value considered in our final model grid, previous runs using coarsely sampled grids showed no sign of convergence for large values of $c$. Similarly, the marginalized $\chi^{2}$ curve for $f_{\text {DM }}$ is essentially flat, and all $f_{\text {DM }}$ values sampled by our grid $\left(50 \leq f_{\mathrm{DM}} \leq 1000\right)$ are allowed within $3 \sigma$. The best-fit value found for $f_{\mathrm{DM}}$ is the minimum value considered in our final grid, but we also ran models without a dark halo and found a significantly worse fit, such that the $\chi^{2}$ increased by 123 relative to the best-fit model with a dark halo. Thus, a dark halo is required to match the observed kinematics, but its properties cannot be pinned down with the current datasets and we do not present any further details associated with the dark halo parameters.

\subsection{Error Budget}

The formal model fitting uncertainties quoted in the previous section are the statistical errors associated with the dynamical models, however systematic effects can have a significant impact on the inferred black hole mass and mass-tolight ratio. In this section, we evaluate some common sources of uncertainty that are not already incorporated into the statistical errors, such as those associated with the assumed form of the dark matter halo, the adopted inclination angle, the number of orbits used in the models, the NIFS PSF model, details associated with the extraction of the NIFS kinematics, and the symmetrization of the input kinematics. 

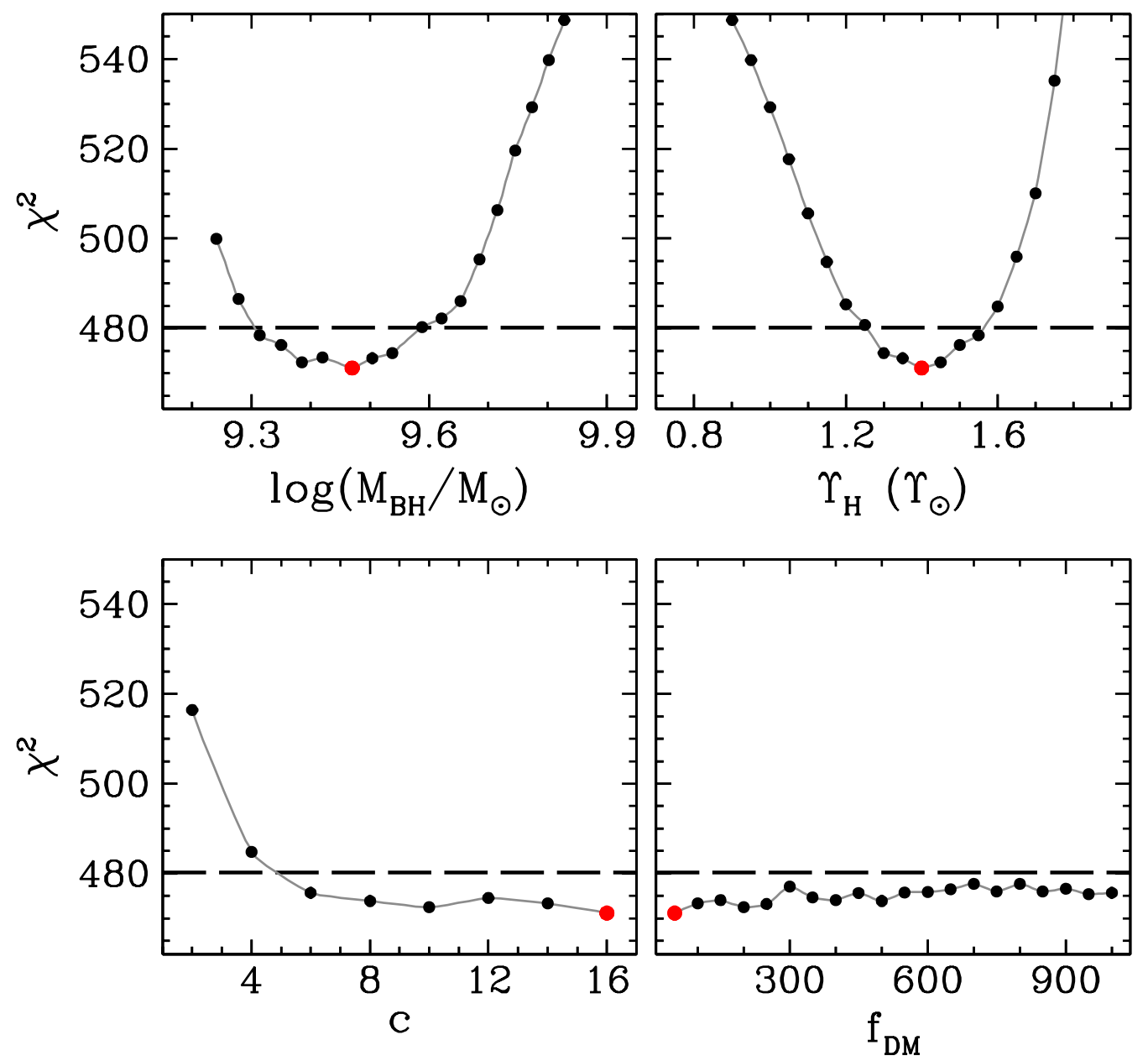

FIG. 4.- The plots summarize the results of the stellar dynamical models run with $i=83^{\circ}$ and an NFW dark matter halo. The $\chi^{2}$ is shown as a function of $M_{\mathrm{BH}}$ (top left), $\Upsilon_{H}$ (top right), $c$ (bottom right), and $f_{\mathrm{DM}}$ (bottom left) after marginalizing over the other three parameters. The red point denotes the best-fit model, and the dashed line depicts where minimum $\chi^{2}$ has increased by 9 , which corresponds to the statistical $3 \sigma$ uncertainties. The uncertainty in $M_{\mathrm{BH}}$ and $\Upsilon_{H}$ due to systematic effects is significantly larger than that statistical $1 \sigma$ error (see Section 7.1). Thus, we do not plot the statistical $1 \sigma$ confidence level, as it is not representative of the range of possible parameter values. The $M_{\mathrm{BH}}$ and $\Upsilon_{H}$ parameters are well constrained despite the large uncertainties associated with the dark halo parameters.

Dark Matter Halo: Previous work has clearly shown that the dark halo could be an important component in the stellar dynamical models due to the degeneracy between the dark halo and stellar mass-to-light ratio, which in turn is also degenerate with the black hole (e.g., Gebhardt \& Thomas 2009; Schulze \& Gebhardt 2011; Rusli et al. 2013). If $r_{\text {sphere }}$ is spatially well resolved, then the degeneracy between the black hole and mass-to-light ratio can be mitigated, and the exclusion of a dark halo will have minimal impact on the inferred $M_{\mathrm{BH}}$. In contrast, if $r_{\text {sphere }}$ is not very well resolved and a dark halo is not included in the stellar dynamical models, then $\Upsilon$ will be artificially elevated to account for the missing mass at large radii. Since $\Upsilon$ is taken to be constant with radius, a smaller $M_{\mathrm{BH}}$ is then required to fit the observed central kinematics.

When generating a grid of models without a dark halo for NGC 1271 , and assuming $i=83^{\circ}$ to match our fiducial model presented in Section 7 , we measure $M_{\mathrm{BH}}=1.4 \times 10^{9} M_{\odot}$ and $\Upsilon_{H}=1.85 \Upsilon_{\odot}$. In other words, the inferred $M_{\mathrm{BH}}$ is underestimated by a factor of $\sim 2$ when a dark halo is excluded from the modeling. We note that when fitting dynamical models without a dark halo to only the small-scale NIFS kinematics, we recover very similar parameter values as those found from our fiducial model, with $M_{\mathrm{BH}}=2.9 \times 10^{9} M_{\odot}$ and $\Upsilon_{H}=1.45 \Upsilon_{\odot}$. Moreover, we tested how the assumed shape of the dark halo affects $M_{\mathrm{BH}}$. Our fiducial model was calculated assuming a spherical NFW halo, but another common form is a halo with a cored logarithmic potential (Binney \& Tremaine 1987; Thomas et al. 2005), given by

$$
\rho_{\mathrm{DM}}(r)=\frac{V_{c}^{2}}{4 \pi G} \frac{3 r_{c}^{2}+r^{2}}{\left(r_{c}^{2}+r^{2}\right)^{2}} .
$$

The parameters $V_{c}$ and $r_{c}$ are the asymptotic circular velocity and radius within which the dark matter density is constant. Thus, the halo from a logarithmic potential yields smaller densities at small radii compared the the NFW halo. We constructed models with a dark halo from a cored logarithmic potential, sampling $9.2 \leq \log \left(M_{\mathrm{BH}} / M_{\odot}\right) \leq 10.2,0.5 \leq \Upsilon_{H} \leq 2.0$ $\Upsilon_{\odot}, 100 \leq V_{c} \leq 700 \mathrm{~km} \mathrm{~s}^{-1}$, and $1 \leq r_{c} \leq 32 \mathrm{kpc}$. We recovered similar results to the models with an NFW halo, namely that $M_{\mathrm{BH}}=3.2 \times 10^{9} M_{\odot}$ and $\Upsilon_{H}=1.35 \Upsilon_{\odot}$, corresponding to a $7 \%$ increase in $M_{\mathrm{BH}}$ and a $4 \%$ decrease in $\Upsilon_{H}$ compared to the fiducial model. Although the black hole mass is sen- 


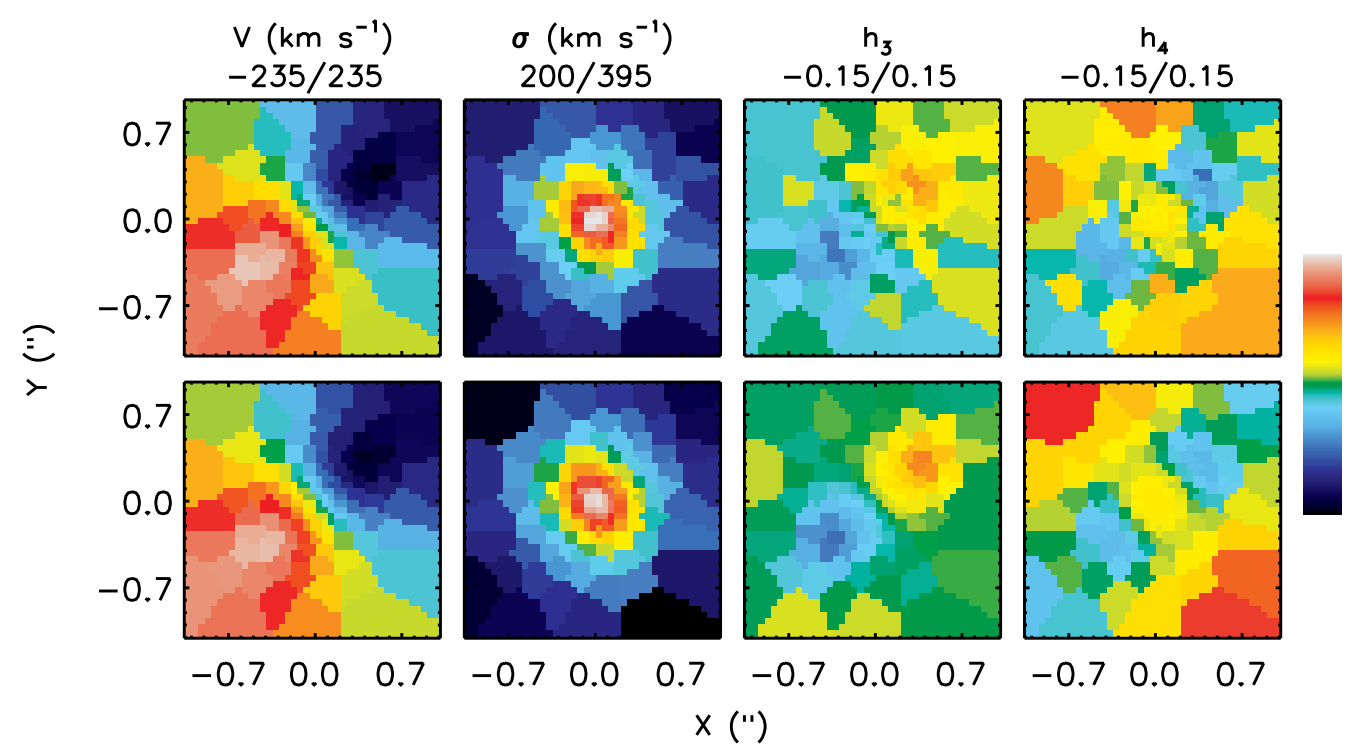

FIG. 5. - The bi-symmetrized NIFS kinematics for NGC 1271 (top) are compared to the best-fit model predictions (bottom), where $M_{\mathrm{BH}}=3.0 \times 10^{9} M_{\odot}$ and $\Upsilon_{H}=1.40 \Upsilon_{\odot}$. The same scaling, shown by the color bar on the right with the minimum and maximum values given at the top of the maps, is used to plot the data and model. The NIIFS obscrvations shew that the galaxy is rapidly rotating with a peak in the velocity dispersion at the nueleus. An anti conriclation between $h_{3}$ and $V$ is found, as is expected for galaxies with axial symmetry. The blue-shiited side of the radial velocity maj corresponds to the southeast side of the galaxy.

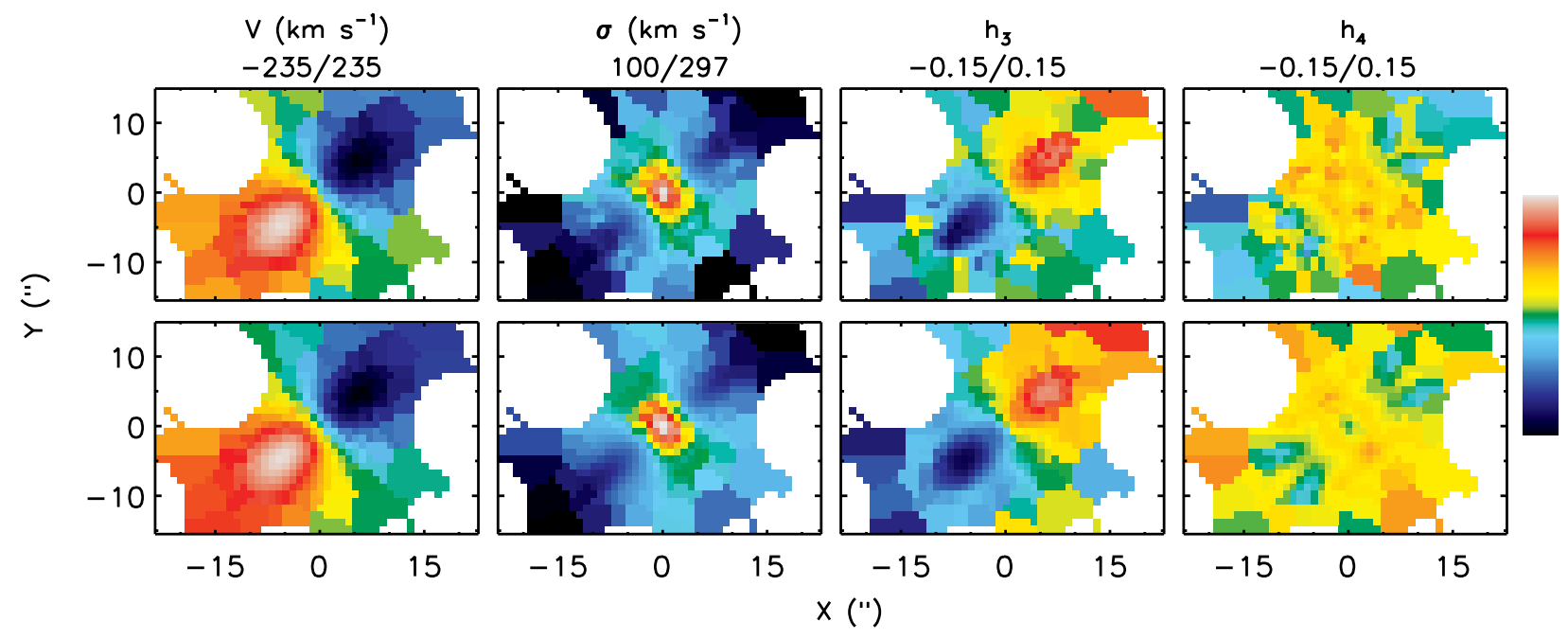

FIG. 6.- The bi-symmetrized PPAK kinematics (top), which extend out to about $4 R_{e}$, are shown along with the predictions from the best-fit stellar dynamical model (bottom); see Figure 5 for description. Kinematic measurements are missing from $x \sim-15^{\prime \prime}, y \sim 10^{\prime \prime}$ and from $x \sim 20^{\prime \prime}, y \sim 0^{\prime \prime}$ due to the presence of foreground objects, which were masked before extracting the kinematics. The southeast side of the galaxy has blue-shifted radial velocities.

sitive to the inclusion of a dark halo in the stellar dynamical models, the form of the halo has a small impact on $M_{\mathrm{BH}}$. For this reason, being unable to constrain the dark halo parameters is not a concern for the purposes of this paper, as long as reasonable halos are sampled over when constructing the orbit-based models.

Inclination Angle: All of the models presented in this paper assume an axisymmetric shape with an inclination angle of $i=83^{\circ}$. However, we also ran a grid of models that sampled 13 inclination angles from 77 to $89^{\circ}$. This corresponds to the range of angles for which the MGE model in Section 3 can be deprojected. It is computationally expensive to calculate a model grid that samples over $M_{\mathrm{BH}}, \Upsilon_{H}, i, c$, and $f_{\mathrm{DM}}$ simultaneously, so we instead varied the first three parameters while sampling over five NFW halos. The five dark halos were those with the lowest $\chi^{2}$ from the model grid at the beginning of Section 7, and the halos span a range of $c$ and $f_{\mathrm{DM}}$ values $\left(8 \leq c \leq 16\right.$ and $\left.50 \leq f_{\mathrm{DM}} \leq 500\right)$. From this test, we determine that $M_{\mathrm{BH}}=3.3 \times 10^{9} M_{\odot}$, which is within $10 \%$ of the best-fit value in Section 7, and $\Upsilon_{H}=1.40 \Upsilon_{\odot}$, which is the same as the best-fit value in Section 7. Moreover, the best-fit inclination angle was $i=87^{\circ}$, however the angle was not well constrained. All angles between $79^{\circ}$ and $89^{\circ}$ were allowed within the $3 \sigma$ statistical uncertainties. Such behavior is not surprising, and other stellar dynamical work have also found it difficult to infer the inclination angle from 2D line-of-sight 
kinematics (Krajnović et al. 2005; van den Bosch \& van de Ven 2009).

Number of Orbits: The fiducial model presented in Section 7 was calculated using orbits that covered 29 equipotential shells with 8 angular and 8 radial values at each energy. When accounting for the orbital dithering, this translates into a total of 696,000 orbits. We also tested the effect on $M_{\mathrm{BH}}$ and $\Upsilon_{H}$ when the number of orbits is about doubled, such that 37 equipotential shells with 10 angular and 10 radial values at each energy are used. Again 125 orbits with adjacent starting positions were bundled together, resulting in $1,387,500$ orbits. Due to a large increase in computational time for a single model, we constructed a model grid that samples over $M_{\mathrm{BH}}, \Upsilon_{H}$, and the top five NFW halos from Section 7. We found no change in the best-fit $M_{\mathrm{BH}}$ or $\Upsilon$ values compared to the fiducial model.

NIFS PSF: The NIFS PSF was measured by comparing the MGE model of the HST image to the collapsed NIFS data cube. While this approach is commonly used in black hole mass measurement work (e.g., Krajnović et al. 2009; Seth et al. 2010; Walsh et al. 2012), estimation of the PSF from $\mathrm{AO}$ observations is notoriously difficult due to the constantly changing quality of the AO correction and the combination of data cubes from multiple nights. Therefore, we also estimated the PSF in a different manner in order to assess how strongly the adopted NIFS PSF affects $M_{\mathrm{BH}}$. Utilizing the NIFS observations of the tip-tilt star, we fit the sum of four concentric, circular 2D Gaussians to the collapsed NIFS data cube using Galfit. We found that the PSF is best described by Gaussians

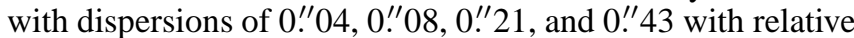
weights of $0.08,0.36,0.26$, and 0.30 . A four-component PSF model provided a significantly better fit to the collapsed data cube than a simpler two-component model, and the residuals between the four Gaussian model and the data have a standard deviation of just $9 \%$ out to a radius of $1^{\prime \prime}$. The PSF is typical of what one would expect from the Gemini AO system (e.g., Gebhardt et al. 2011; Onken et al. 2014), and the quality of the $\mathrm{AO}$ correction is better than that implied by the PSF adopted in the fiducial model. This is not completely surprising as the NIFS observations of the star used the star itself for tiptilt corrections, whereas the observations of the galaxy were made off-axis, using the star for guiding. Nonetheless, calculating stellar dynamical models using this better PSF and comparing to the results using the poorer PSF should cover the range of possible black hole masses due to the uncertainty in the NIFS PSF. We calculated models using the new fourGaussian PSF and further assumed that the center of the NIFS spaxel with the largest flux coincides with the galaxy nucleus. We varied $M_{\mathrm{BH}}$ and $\Upsilon_{H}$, while sampling over the top five NFW halos from Section 7 , and found $M_{\mathrm{BH}}=2.7 \times 10^{9} M_{\odot}$ and $\Upsilon_{H}=1.45 \Upsilon_{\odot}$. Therefore, the black hole mass and massto-light ratio change by $10 \%$ and $4 \%$ compared to the fiducial values in Section 7.

Measuring the NIFS Kinematics: We measured the NIFS kinematics with pPXF using a second degree additive Legendre polynomial and a second degree multiplicative polynomial to correct for shape differences between the LOSVDbroadened optimal stellar template and the observed galaxy spectrum. We selected this continuum correction because it was one of the simplest models that still provided a good fit to the data, and produced kinematics that were in good agreement with those measured using combinations of degree $0-3$ additive/multiplicative polynomials, with the exception of the lowest order polynomials. Since the continuum has been previously divided out of the the spectral templates in the NIFS library, but not removed from the observed galaxy spectra, using the lowest order polynomials (combinations of degree $0-1$ additive/multiplicative polynomials) produced visibly poor spectral fits.

We also constructed a new library containing stellar templates that have not been continuum-corrected and thus have a very similar shape as the galaxy spectra. We retrieved $K$-band NIFS observations from the Gemini archive of stars that are part of the NIFS Spectral Template Library and two stars observed under program GN-2010A-Q-112. These twelve stars are K0 - M5 giants, a K5 supergiant, and an M0 supergiant. We reduced the observations following the main procedure outlined in Section 2.2, with the additional steps of extracting a one-dimensional spectrum, rebinning to a common wavelength range and sampling, and shifting the stars to rest. With this new template library, we are able to obtain good fits to the galaxy spectra using low-order polynomials with pPXF.

In order to examine possible effects on $M_{\mathrm{BH}}$ and $\Upsilon_{H}$ due to uncertainties associated with the choice of the pPXF polynomial degree, we fit dynamical models to the NIFS kinematics extracted using the new stellar template library and an additive constant, along with the PPAK kinematics presented in Section 4.2. We sampled over $M_{\mathrm{BH}}, \Upsilon_{H}$, and the top five NFW halos from Section 7 , finding $M_{\mathrm{BH}}=2.4 \times 10^{9} M_{\odot}$ and $\Upsilon_{H}=1.45 \Upsilon_{\odot}$. This corresponds to a change of $20 \%$ and $4 \%$ from the best-fit black hole mass and mass-to-ratio from the fiducial model. These results are likely representative of a maximum change in best-fit parameter values, as using this particular continuum correction with the new stellar template library resulted in the largest number of bins with kinematics inconsistent at the $1 \sigma$ level (all bins were consistent at the $2 \sigma$ level) compared to the NIFS kinematics from Section 4.1.

Symmetrizing the Kinematics: The NIFS and PPAK kinematics were bi-symmetrized prior to using them as inputs into the stellar dynamical modeling code. We used the method outlined in van den Bosch \& de Zeeuw (2010), which averages the measurements of a GH moment in a four-fold symmetric manner around the minor and major axes. During the averaging of the measurements for a single $\mathrm{GH}$ moment, the kinematic error of the bin and the fraction of flux a spaxel contributes to that bin are taken into account. Such modifications to the input kinematics and their errors are a common way in which to reduce observational noise, and often kinematics are bi-symmetrized in order to obtain reasonable results from an axisymmetric modeling code and are point-symmterized for use with a triaxial modeling code. However, symmterization routines are never perfect, as discussed in van den Bosch \& de Zeeuw (2010). We therefore tested how symmetrization affects $M_{\mathrm{BH}}$ and $\Upsilon_{H}$ by running additional models with kinematics that were point-symmetrized. We find that the black hole mass increases to $3.6 \times 10^{9} M_{\odot}$, or by $20 \%$ of the fiducial value, and the $H$-band mass-to-light ratio decreases to $1.35 \Upsilon_{\odot}$, or $4 \%$ of the fiducial value from Section 7 .

Summary: We derive the final range of black hole masses and mass-to-light ratios for NGC 1271 by adding in quadrature the formal model fitting $1 \sigma$ uncertainty from Section 7 and the additional sources of systematic uncertainty above. Ultimately, we determine that $M_{\mathrm{BH}}=\left(3.0_{-1.1}^{+1.0}\right) \times 10^{9} M_{\odot}$ and $\Upsilon_{H}=1.40_{-0.11}^{+0.13} \Upsilon_{\odot}$. The dominant source of systematics for NGC 1271 are those associated with the continuumcorrection model used to extract the NIFS kinematics and the 
symmetrization of the kinematics. Both affect $M_{\mathrm{BH}}$ and $\Upsilon_{H}$ at the $20 \%$ and $4 \%$ level, respectively.

\subsection{Other Considerations}

In addition to examining possible sources of systematic uncertainty and incorporating the effects into the final error budget, we ran other tests to assess the robustness of the NGC $1271 M_{\mathrm{BH}}$ measurement. We describe these tests below.

Nuclear Dust Disk: A dust disk is present at the center of NGC 1271, and is visible in the F814W WFC3 image. However, dust doesn't appear to be significant in the near-infrared imaging and in the NIFS data cube. We tested constructing a new MGE of the F160W image after excluding the dust disk by using the F814W image as guide for generating the mask. We ran dynamical models using the modified MGE, following the procedure in Section 6.1 and sampling over the top five NFW halos from Section 7. We found best-fit values of $M_{\mathrm{BH}}=3.2 \times 10^{9} M_{\odot}$ and $\Upsilon_{H}=1.35 \Upsilon_{\odot}$, which is well within the final uncertainties adopted for NGC 1271 discussed in Section 7.1.

Stellar Mass-to-Light Ratio Variation: Our dynamical models assume that $\Upsilon_{H}$ remains constant with radius. In order to determine whether there is an obvious change in stellar population, we generated an MGE of the F814W image following the methods described in Section 3. During the fit, we masked out the nuclear dust disk and foreground objects in the F814W image, and we accounted for the PSF using a bright, isolated star in the image. From the MGE models of the F814W and F160W images, we don't see evidence for color gradients, finding that the color changes by at most 0.16 mag from $0 . / 2$ to $24^{\prime \prime}$.

Although NGC 1271 exhibits a fairly uniform color, we further examined dynamical models that are fit to only the NIFS kinematics. Given the limited radial extent of the NIFS kinematics, which extend out to a radius of $\sim 1^{\prime \prime}$, or $\sim 390$ pc, systematics associated with mass-to-light ratio gradients (and dark matter halos) are mitigated. When fitting to only the NIFS kinematics, we recover consistent results to those presented in 7.1, where $M_{\mathrm{BH}}=\left(3.5_{-1.0}^{+0.4}\right) \times 10^{9} M_{\odot}$ and $\Upsilon_{H}=$ $1.30_{-0.07}^{+0.25} \Upsilon_{\odot}(1 \sigma$ uncertainties $)$. We show contours of $\chi^{2}$ as a function of black hole mass and mass-to-light ratio for this NIFS-only model grid in Figure 7.

PPAK Kinematics: When measuring the stellar kinematics from the PPAK data, we masked out spectral regions containing possible emission lines and sky residuals, as can be seen in Figure 3 . In order to verify that our choice of a spectral mask does not bias the kinematic measurements and inferred black hole mass, we decreased the number and width of the excluded wavelength regions. We ran dynamical models using the modified PPAK kinematics while sampling over the top five dark matter halos in Section 7. We measured $M_{\mathrm{BH}}=3.2 \times 10^{9} M_{\odot}$ and $\Upsilon_{H}=1.35 \Upsilon_{\odot}$, which is within the final uncertainties given for NGC 1271 in Section 7.1.

Also, the observed velocity dispersion of the PPAK kinematics presented in Section 4.2 drops below the instrumental resolution in many of the spatial bins located $\gtrsim 10^{\prime \prime}$ away from the nucleus. While care was taken to homogenize the line spread function to a common value such that there was no variation with wavelength or fiber position, measuring dispersions well below the instrumental resolution is a difficult task. We therefore also tested the effect on $M_{\mathrm{BH}}$ and $\Upsilon_{H}$ when excluding the spatial bins in which the dispersion was below $150 \mathrm{~km} \mathrm{~s}^{-1}$. When calculating dynamical models that fit to

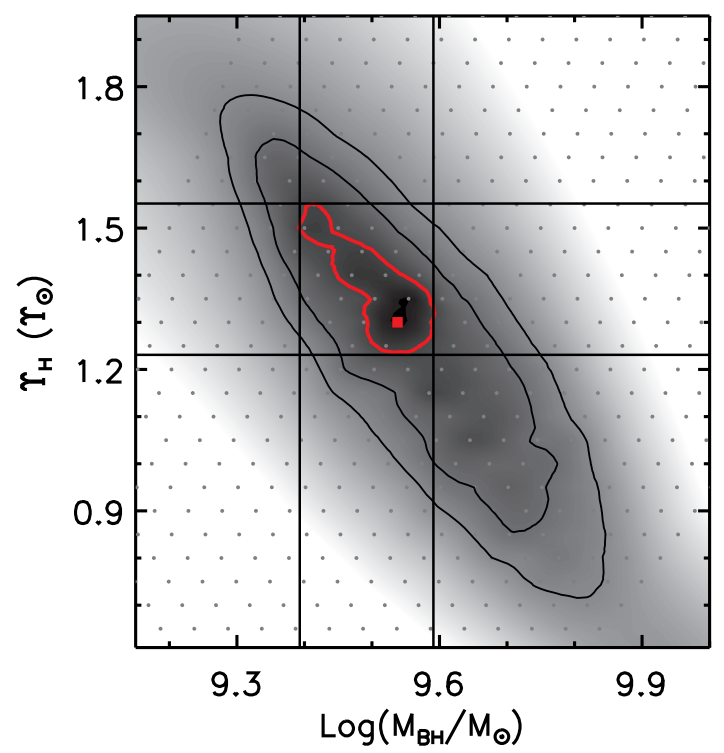

FIG. 7.- Contours of $\chi^{2}$ are shown as a function of black hole mass and $H$-band mass-to-light ratio for the case when dynamical models are fit to just the NIFS kinematics. At each gray point a model was calculated, and the red square denotes the best-fit model. The red contour and two black contours signify where $\chi^{2}$ has increased by 1,4 , and 9 from the minimum. Thus, the vertical lines show the $1 \sigma$ uncertainties on $M_{\mathrm{BH}}$ and the horizontal lines give the $1 \sigma$ uncertainties for $\Upsilon_{H}$ when marginalizing over the other parameters.

the adjusted PPAK kinematics and that sample over the top five dark matter halos in Section 7, we find no change from the best-fit values presented in Section 7.

\section{DISCUSSION}

NGC 1271 harbors a black hole with $M_{\mathrm{BH}}=\left(3.0_{-1.1}^{+1.0}\right) \times$ $10^{9} M_{\odot}$ and has a stellar mass-to-light ratio of $\Upsilon_{H}=$ $1.40_{-0.11}^{+0.13} \Upsilon_{\odot}$. We note that the final uncertainty on the black hole mass we use is comparable to the formal $3 \sigma$ statistical uncertainty. Some (Cappellari et al. 2009; Krajnović et al. 2009; Emsellem 2013) have suggested $3 \sigma$ statistical errors should be used in place of $1 \sigma$ errors as a conservative way in which to account for the effect of unknown systematics on $M_{\mathrm{BH}}$. With a black hole mass of $3.0 \times 10^{9} M_{\odot}$ and adopting $276 \mathrm{~km} \mathrm{~s}^{-1}$ for the bulge stellar velocity dispersion (see Section 8.2), $r_{\text {sphere }}=0$ !' 44 . Thus, the NIFS observations have resolved the black hole sphere of influence. Below we discuss the galaxy's orbital structure and place the galaxy on the $M_{\mathrm{BH}}$-host galaxy relations.

\subsection{Orbital Structure}

In addition to determining the mass of the black hole in NGC 1271, the Schwarzschild models provide information about the galaxy's orbital structure. Using our best-fit model in Section 7, we show the ratio $\sigma_{r} / \sigma_{t}$ as a function of radius in Figure 8. The tangential velocity dispersion is defined as $\sigma_{t}^{2}=\left(\sigma_{\phi}^{2}+\sigma_{\theta}^{2}\right) / 2$, and $(r, \theta, \phi)$ are the usual spherical coordinates. We find that NGC 1271 is roughly isotropic at all radii covered by our kinematic measurements, deviating by at most $30 \%$ from $\sigma_{r} / \sigma_{t}=1$, but we observe a trend in which $\sigma_{r} / \sigma_{t}$ declines at radii outside the black hole sphere of influence. As expected, short-axis tube orbits dominate in this oblate system, making up more than $85 \%$ of the orbits at all radii. Long-axis tube orbits, which are important for triaxial and prolate systems, are negligible, while the fraction of box 


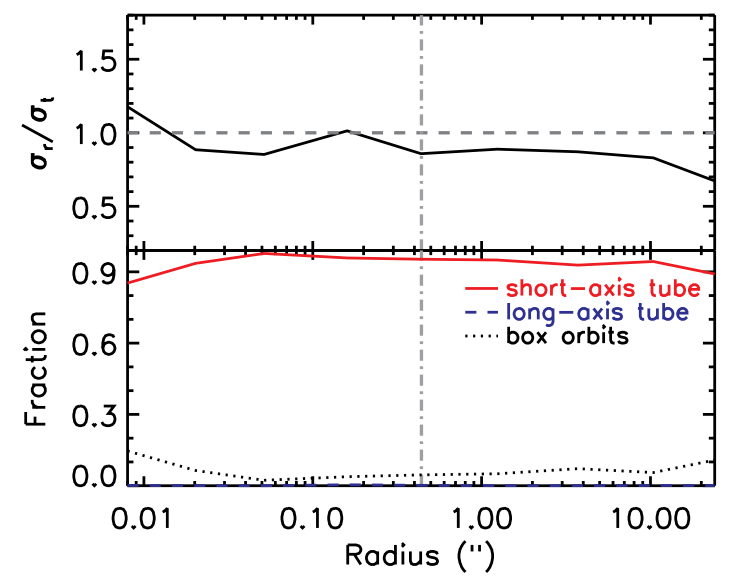

FIG. 8.- NGC 1271's orbital structure, as inferred from the best-fit dynamical model, is shown. The anisotropy (top) and orbit type (bottom) are displayed with radius over the range covered by the NIFS and PPAK kinematic measurements. The horizontal dashed gray line denotes the isotropic case and the vertical dot-dashed gray line shows the black hole sphere of influence. NGC 1271 is roughly isotropic at all radii and is dominated by short-axis tube orbits as is expected for oblate systems.

orbits increases at small radii but still make up only $15 \%$ of the orbits near the nucleus.

Furthermore, we use our best-fit stellar dynamical model to examine the mass distribution as a function of average radius, $\bar{r}$, and spin, $\bar{\lambda}_{z}$, of the orbits, where $\bar{\lambda}_{z}=\bar{J}_{z} \times(\bar{r} / \bar{\sigma})$. Here, $\bar{J}_{z}$ is the average angular momentum along the $z$-direction and $\bar{\sigma}$ is the average second moment of the orbit. NGC 1271 shows several dynamical components, as can be seen in Figure 9, including a clear non-rotating bulge (with $-0.2<\bar{\lambda}_{z}<0.2$ ), a highly co-rotating component (with $\bar{\lambda}_{z} \sim 0.5$ ), and a maximally co-rotating component (with $0.8<\bar{\lambda}_{z}<1.0$ ). The bulge component accounts for $12 \%$ of the mass, whereas the rotating components total $75 \%$ of the mass within the radial extent of the kinematic measurements. Qualitatively these components agree with the classification of NGC 1271 as a fastrotating S0 galaxy with a classical bulge.

\subsection{Black Hole - Host Galaxy Relations}

Although the dynamical decomposition from the best-fit stellar dynamical model presented above can be used to place NGC 1271 on the $M_{\mathrm{BH}}$ - bulge relationships, we follow the more common approach of carrying out a photometric decomposition to determine the galaxy's bulge luminosity and bulge effective radius. Using Galfit, we find a single Sérsic component fit is an insufficient description of the galaxy, with the percent difference between the model and data reaching as high as $60 \%$. The fit is significantly improved with the addition of one or two other Sérsic components, and in the later case the percent difference between the model and data is under $15 \%$. In Table 4, we present the best-fit parameters of Galfit models with one, two, and three Sérsic components, as well as the F160W luminosity for each component. The three-component model provides the best match to the HST image, but it is difficult to unambiguously identify a "bulge" component because the components all have rather low Sérsic indices. Therefore, we conservatively assume that the innermost component of the three-component model provides a lower limit on the bulge luminosity and effective radius, while the innermost component of the two-component model gives an upper limit. This yields a $K$-band bulge luminosity

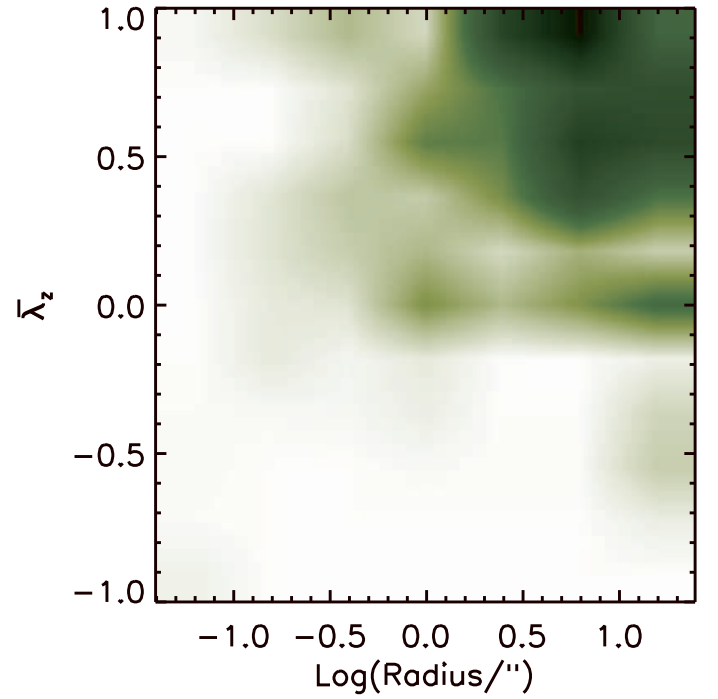

FIG. 9.- The mass distribution is plotted as a function of average spin and radius of the orbits for the region covered by our kinematics measurements. The dynamical decomposition utilizes the orbital weights from our best-fit stellar dynamical model and shows distinct non-rotating bulge $\left(-0.2<\bar{\lambda}_{z}<\right.$ $0.2)$ and rotating $\left(\bar{\lambda}_{z}>0.2\right)$ disk components.

TABLE 4

GALFIT MODELS

\begin{tabular}{cccccc}
\hline \hline $\begin{array}{c}\text { Component } \\
(1)\end{array}$ & $\begin{array}{c}m_{H} \\
(2)\end{array}$ & $\begin{array}{c}L_{H}\left(L_{\odot}\right) \\
(3)\end{array}$ & $\begin{array}{c}R_{e}\left({ }^{\prime \prime}\right) \\
(4)\end{array}$ & $\begin{array}{c}n \\
(5)\end{array}$ & $\begin{array}{c}q^{\prime} \\
(6)\end{array}$ \\
\hline 1 & 10.72 & $7.7 \times 10^{10}$ & 5.56 & 4.78 & 0.41 \\
\hline 1 & 10.95 & $6.2 \times 10^{10}$ & 5.23 & 6.54 & 0.54 \\
2 & 12.47 & $1.5 \times 10^{10}$ & 6.63 & 0.93 & 0.20 \\
\hline 1 & 12.39 & $1.6 \times 10^{10}$ & 0.61 & 2.12 & 0.68 \\
2 & 11.80 & $2.8 \times 10^{10}$ & 5.24 & 1.17 & 0.27 \\
3 & 11.90 & $2.6 \times 10^{10}$ & 10.46 & 1.30 & 0.62 \\
\hline
\end{tabular}

NoTE. - Column (1) shows the Sérsic component number, column (2) provides the F160W apparent magnitude in the Vega system, not yet corrected for galactic extinction, column (3) gives the luminosity after a correction of 0.085 for galactic extinction and assuming an absolute solar magnitude of 3.33, column (4) is the effective radius, column (5) gives the Sérsic index, and column (6) lists the projected axis ratio.

of $(1.9-7.2) \times 10^{10} L_{\odot}$ when correcting for galactic extinction using the Schlafly \& Finkbeiner (2011) WFC3 F160W value of 0.085 , assuming an $H-K$ color of 0.2 (Vazdekis et al. 1996), and a $K$-band solar absolute magnitude of 3.29. Alternatively, the bulge has a mass of $(2.3-8.7) \times 10^{10} M_{\odot}$ when applying the best-fit mass-to-light ratio from our dynamical models to the luminosities in Table 4 . The corresponding bulge effective radius ranges between $0 .{ }^{\prime \prime} 6$ and 5." 2 (or $0.2-2.0 \mathrm{kpc}$ ).

In order to determine the bulge stellar velocity dispersion for NGC 1271, we use the best-fit stellar dynamical model from Section 7 and predict the luminosity-weighted second moment within a circular aperture whose radius equals the galaxy's bulge effective radius, following the approach used by van den Bosch et al. (2012). Due to the uncertainty in the bulge effective radius for NGC 1271, we measure the effective stellar velocity dispersion $\left(\sigma_{e, \text { bul }}\right)$ for three different bulge effective radii corresponding to the largest $R_{e}$ estimate from the Galfit decomposition, the smallest estimate of $R_{e}$, and the average of the two. Additionally, some previous black hole 
studies have chosen to exclude data within $r_{\text {sphere }}$ when determining $\sigma_{e \text {,bul }}$ because the stellar kinematics are under the direct influence of the black hole in this region (e.g., Gebhardt et al. 2011; McConnell \& Ma 2013). When excluding the region within $r_{\text {sphere }}$, we find effective stellar velocity dispersions of $\sigma_{e, \text { bul }}=272 \mathrm{~km} \mathrm{~s}^{-1}, \sigma_{e, \text { bul }}=276 \mathrm{~km} \mathrm{~s}^{-1}$, and $\sigma_{e, \text { bul }}=349 \mathrm{~km} \mathrm{~s}^{-1}$, whereas when the region within $r_{\text {sphere }}$ is included we measure $\sigma_{e, \text { bul }}=285 \mathrm{~km} \mathrm{~s}^{-1}, \sigma_{e \text {,bul }}=294 \mathrm{~km} \mathrm{~s}^{-1}$, and $\sigma_{e, \text { bul }}=358 \mathrm{~km} \mathrm{~s}^{-1}$, for bulge effective radii of 5.'2, 2.'9, and $0 . \prime 6$, respectively. As a comparison, the HET Massive Galaxy Survey reports a central velocity dispersion of $317 \mathrm{~km}$ $\mathrm{s}^{-1}$ (van den Bosch et al. 2015), which is the observed stellar velocity dispersion within a 3. . 5 aperture from the major axis long-slit data.

Figure 10 shows the location of NGC 1271 on the most recent versions of the $M_{\mathrm{BH}}-\sigma_{\star}$ and $M_{\mathrm{BH}}-L_{\text {bul }}$ relations by Kormendy \& Ho (2013). For the purposes of placing NGC 1271 on the $M_{\mathrm{BH}}-\sigma_{\star}$ correlation, we use $\sigma_{\star}=276 \mathrm{~km} \mathrm{~s}^{-1}$ with uncertainties that include the $\sigma_{e \text {,bul }}$ measurements made for bulge effective radii of 5." 2 and $0 . " 6$ when excluding data within $r_{\text {sphere. }}$ When placing NGC 1271 on the $M_{\mathrm{BH}}-L_{\mathrm{bul}}$ relation, we set the faint end of the bulge luminosity error bar assuming the luminosity of the innermost component of the three-component Sérsic fit to the HST image and the high end of the error bar assuming the luminosity of the innermost component of the two-component Sérsic fit. We adopt a $K$ band bulge luminosity of $4.6 \times 10^{10} L_{\odot}$, which is the midpoint of the range of possible bulge luminosities.

We find that NGC 1271 consistent with the $M_{\mathrm{BH}}-\sigma_{\star}$ relation, but is an order of magnitude above the black hole mass prediction from the $M_{\mathrm{BH}}-L_{\text {bul }}$ correlation. In order to demonstrate that the black hole in NGC 1271 must be larger than that expected from $M_{\mathrm{BH}}-L_{\mathrm{bul}}$, in Figure 11 we present the NIFS observations of the velocity dispersion and $h_{4}$ along with the predictions from the best-fitting model with a black hole mass of $3.0 \times 10^{9} M_{\odot}$ and a $4.7 \times 10^{8} M_{\odot}$ black hole. The $4.7 \times 10^{8} M_{\odot}$ black hole is expected from $M_{\mathrm{BH}}-L_{\mathrm{bul}}$ when conservatively using the galaxy's total $K$-band luminosity of $8.9 \times 10^{10} L_{\odot}$, which is derived from the single Sérsic fit to the $H S T$ image, after correcting for galactic extinction and assuming a $H-K=0.2$. Clear differences between the kinematic predictions and the observations can be seen by eye. The best-fit model with $M_{\mathrm{BH}}=3.0 \times 10^{9} M_{\odot}$ is able to nicely reproduce the sharp rise in the velocity dispersion and the slight peak in $h_{4}$ at the nucleus, while the less massive black hole predicted from $M_{\mathrm{BH}}-L_{\text {bul }}$ fails to do so.

NGC 1271 has an apparent ellipticity of $\epsilon=0.6$ and a specific stellar angular momentum of $\lambda_{R} \equiv$ $\langle R|V|\rangle /\left\langle R \sqrt{V^{2}+\sigma^{2}}\right\rangle=0.5$ within one effective radius based on the PPAK data. Here, $R, V$, and $\sigma$ are the radius, velocity, and velocity dispersion and the brackets denote a luminosity weighted average (Emsellem et al. 2007). Using the dividing line between slow and fast rotators from the ATLAS $^{3 \mathrm{D}}$ survey, such that fast rotators have $\lambda_{R} \geq 0.31 \times \sqrt{\epsilon}$ (Emsellem et al. 2011), NGC 1271 falls well within in this fast rotator regime.

NGC 1271 appears similar to the other compact galaxies NGC 1277, NGC 1332, NGC 4342, NGC 4486B, and M60UCD1. All six of these early-type galaxies have small sizes, are rotating, show large stellar velocity dispersions for their luminosities, and have black holes that are too massive for their host galaxy's bulge luminosity. The black holes, how- ever, are consistent with $M_{\mathrm{BH}}-\sigma_{\star}$ given the intrinsic scatter of the relation. In the case of M60-UCD1, Seth et al. (2014) suggest that the ultracompact dwarf galaxy (UCD) was once the nucleus of a larger galaxy that has since been tidally stripped by the giant elliptical M60, whose center lies at a projected distance of just $6.6 \mathrm{kpc}$ away from the UCD. While tidal stripping is a natural explanation for the presence of an overmassive black hole, in the case of NGC 1271, we do not see signs of active stripping in the HST image. The isophotes appear extremely regular, and no massive galaxies immediately neighbor NGC 1271 like in the case of M60-UCD1. NGC 1271 is $\sim 270 \mathrm{kpc}$ in projection from the BCG of Perseus. Further evidence could come from counting the number of globular clusters, as the galaxy would be stripped of its globular clusters first. While NGC 1271 appears not to have been stripped with our current data, we cannot rule out an event in the distant past.

Interestingly, the behavior of the compact, high-dispersion galaxies being consistent with $M_{\mathrm{BH}}-\sigma_{\star}$ but being large positive outliers on $M_{\mathrm{BH}}-L_{\mathrm{bul}}$ could be in conflict with recent observations of BCGs, which instead may hint that black hole mass becomes independent of $\sigma_{\star}$ at high black hole mass while the $M_{\mathrm{BH}}-L_{\mathrm{bul}}$ correlation remains unchanged at large luminosities (McConnell \& Ma 2013; Kormendy \& Ho 2013). Clearly, more compact, high-dispersion galaxies and BCGs/giant ellipticals need to be examined. There could be systematic differences in the scaling relations between the two types of galaxies, thereby imply different mechanisms for black hole growth. Since the compact, high-dispersion galaxies like NGC 1271 look similar to the quiescent $z \sim 2$ red nuggets, they could be relics that somehow avoided the same fate that ultimately produced the giant ellipticals observed today. Perhaps the compact, high-dispersion galaxies are left over from an era when the local black hole scaling relations did not apply and galaxies instead contained overmassive black holes.

\section{CONCLUSION}

To summarize, we obtained AO-assisted Gemini NIFS observations of NGC 1271 to map out the stellar kinematics on scales comparable to the black hole sphere of influence, and large-scale IFU data with PPAK, which are useful for constraining the galaxy's stellar mass-to-light ratio and orbital distribution. Using an HST WFC3 $H$-band image along with the spectral information, we constructed orbit-based stellar dynamical models. We measure $M_{\mathrm{BH}}=\left(3.0_{-1.1}^{+1.0}\right) \times 10^{9} M_{\odot}$ and $\Upsilon_{H}=1.40_{-0.11}^{+0.13} \Upsilon_{\odot}$. The quoted errors combine the $1 \sigma$ model fitting uncertainties with some common sources of systematic uncertainty that affect stellar dynamical models. The black hole in NGC 1271 is at the upper end of the black hole mass distribution $\left(M_{\mathrm{BH}}>1 \times 10^{9} M_{\odot}\right)$. Yet, this compact, rapidly rotating galaxy, with a high stellar velocity dispersion for its luminosity is very different from the giant elliptical galaxies and BCGs that are expected to harbor the most massive black holes in the Universe. Such host galaxy environments have yet to be widely explored on the $M_{\mathrm{BH}}-$ host galaxy relations. With our mass measurement, we find that the black hole is too large for the galaxy's $K$-band bulge luminosity of $\left(4.6_{-2.7}^{+2.6}\right) \times 10^{10} L_{\odot}$, falling an order of magnitude above the expectation from the $M_{\mathrm{BH}}-L_{\text {bul }}$ correlation, but the black hole mass is consistent with expectations from the $M_{\mathrm{BH}}-\sigma_{\star}$ relationship assuming $\sigma_{\star}=276_{-4}^{+73} \mathrm{~km} \mathrm{~s}^{-1}$. This behavior has also been observed in the few other compact 


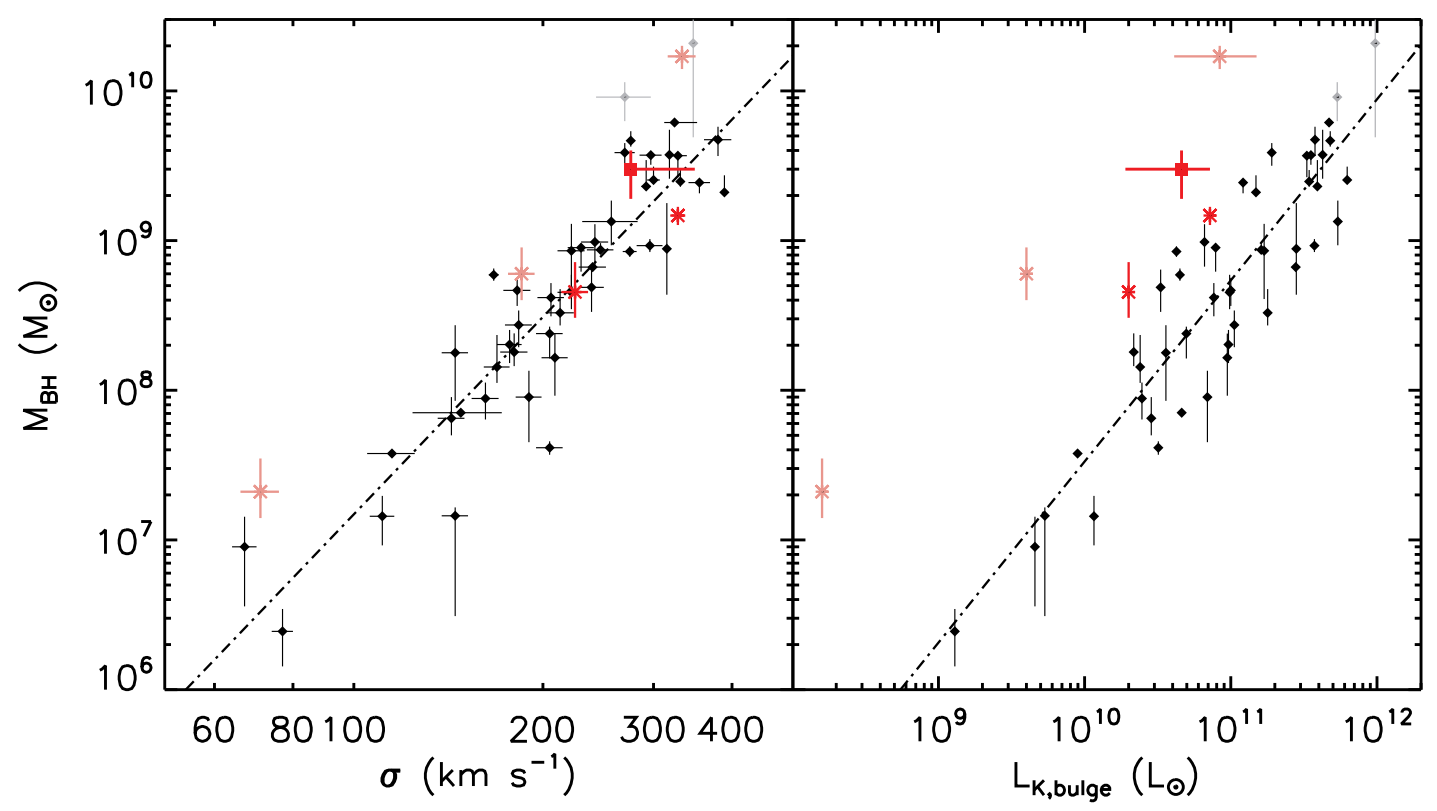

FIG. 10.- NGC 1271 (red filled square) is shown on the black hole - host galaxy relations. The black hole/galaxy measurements (black and gray filled circles) and the fitted relations (dot dashed lines) are taken from Kormendy \& Ho (2013). The compact galaxies that have existing dynamical $M_{\mathrm{BH}}$ measurements are denoted with the red asterisks. These galaxies are generally consistent with $M_{\mathrm{BH}}-\sigma_{\star}$ but are positive outliers from $M_{\mathrm{BH}}-L_{\mathrm{bul}}$. Kormendy \& Ho (2013) did not include the measurements shown in gray and light red when fitting the black hole scaling relations.

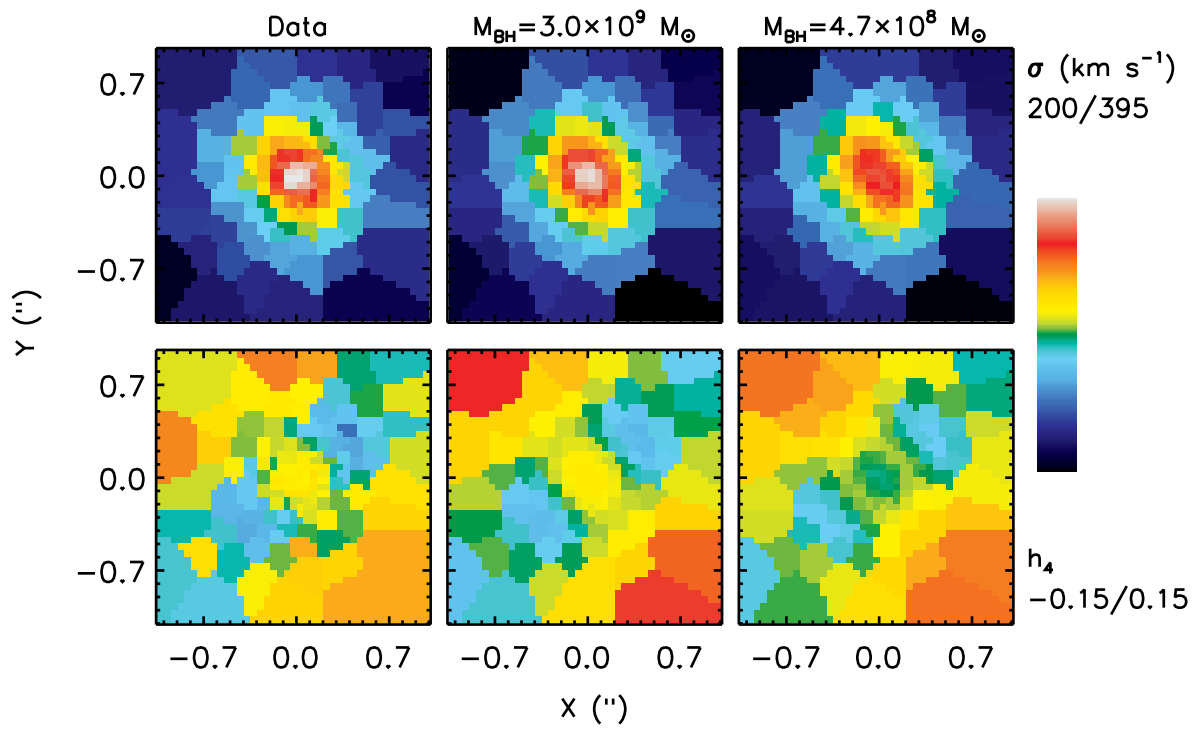

FIG. 11.- The observed velocity dispersion (top) and $h_{4}$ (bottom) measured from the NIFS data (left) is compared to predictions from the best-fit model with $M_{\mathrm{BH}}=3.0 \times 10^{9} M_{\odot}$ (middle) and a model with a $4.7 \times 10^{8} M_{\odot}$ black hole (right), which is the mass predicted from $M_{\mathrm{BH}}-L_{\text {bul }}$ when conservatively adopting the total galaxy luminosity. When generating the $\sigma$ and $h_{4}$ predictions for a $4.7 \times 10^{8} M_{\odot}$ black hole, we sample over $\Upsilon_{H}$ and the top five NFW dark halos from the model grid in Section 7, such that the combination of parameters produces a model with the lowest $\chi^{2}$ for a black hole mass of $4.7 \times 10^{8} M_{\odot}$. The data and model maps are plotted on the same scale, with the ranges given by the color bar to the right and the minimum and maximum values printed at the side of the maps. The best-fit model is able to reproduce the sharp rise in the velocity dispersion and slight peak in $h_{4}$ at the center, while the smaller black hole expected from the $M_{\mathrm{BH}}-L_{\mathrm{bul}}$ correlation is unable to match either of these features. 
galaxies that have dynamical black hole mass measurements to date. Carrying out more black hole mass measurements in similar galaxies using high spatial resolution observations from $H S T$ and $\mathrm{AO}$ is necessary in order to determine if there are systematic differences in the black hole scaling relations between the large ellipticals/BCGs and these compact, highdispersion galaxies. The compact, high-dispersion galaxies could be remnants of the $z \sim 2$ red nugets that for some reason did not evolve into the largest ellipticals observed today, and instead reflect a time when black holes were too large for their bulges. More broadly, additional black hole mass measurements are needed in order to enlarge and better fill in undersampled regions of galaxy parameter space. Targeting such a large and carefully selected sample with high spatial resolution facilities is a natural step toward gaining a more complete census of local black holes and a better understanding of the role that black holes play in galaxy evolution.

J. L. W. has been supported by an NSF Astronomy and Astrophysics Postdoctoral Fellowship under Award No. 1102845. Based on observations obtained at the Gemini Observatory, which is operated by the Association of Universities for Research in Astronomy, Inc., under a cooperative agreement with the NSF on behalf of the Gemini partnership: the National Science Foundation (United States), the National Research Council (Canada), CONICYT (Chile), the Australian Research Council (Australia), Ministério da Ciên- cia, Tecnologia e Inovação (Brazil) and Ministerio de Ciencia, Tecnología e Innovación Productiva (Argentina), under program GN-2012B-Q-51. Also based on observations made with the NASA/ESA Hubble Space Telescope, obtained at the Space Telescope Science Institute, which is operated by the Association of Universities for Research in Astronomy, Inc., under NASA contract NAS 5-26555. These observations are associated with program \#13050. This work is further based on observations collected at the Centro Astronómico Hispano Alemán (CAHA) at Calar Alto, operated jointly by the Max-Planck Institut für Astronomie and the Instituto de Astrofísica de Andalucía (CSIC). The authors acknowledge the Texas Advanced Computing Center (TACC; http://www.tacc.utexas.edu) at The University of Texas at Austin for providing HPC resources that have contributed to the research results reported within this paper. The authors also made use of the grant-funded cyberinfrastructure at Indiana University. This material is based upon work supported by the National Science Foundation under Grant No. CNS0723054, and in part by Lilly Endowment, Inc., through its support for the Indiana University Pervasive Technology Institute, and in part by the Indiana METACyt Initiative. The Indiana METACyt Initiative at Indiana University is also supported in part by Lilly Endowment, Inc. This research has made use of the NASA/IPAC Extragalactic Database which is operated by the Jet Propulsion Laboratory, California Institute of Technology, under contract with NASA.

\section{REFERENCES}

Bender, R., Saglia, R. P., \& Gerhard, O. E. 1994, MNRAS, 269, 785 Bertin, E., \& Arnouts, S. 1996, A\&AS, 317, 393

Binney, J., \& Tremaine, S. 1987, Galactic Dynamics, ed. Binney, J. \& Tremaine, $\mathrm{S}$.

Blom, C., Forbes, D. A., Foster, C., Romanowsky, A. J., \& Brodie, J. P. 2014, MNRAS, 439, 2420

Boccas, M., et al. 2006, Proc. SPIE, 6272, 114

Bogdán, Á., et al. 2012, ApJ, 753, 140

Bureau, M., \& Athanassoula, E. 2005, ApJ, 626, 159

Cappellari, M. 2002, MNRAS, 333, 400

Cappellari, M., \& Copin, Y. 2003, MNRAS, 342, 345

Cappellari, M., \& Emsellem, E. 2004, PASP, 116, 138

Cappellari, M., et al. 2006, MNRAS, 366, 1126

Cappellari, M., Neumayer, N., Reunanen, J., van der Werf, P. P., de Zeeuw,

P. T., \& Rix, H.-W. 2009, MNRAS, 394, 660

Cretton, N., \& van den Bosch, F. C. 1999, ApJ, 514, 704

Dalla Bontà, E., Ferrarese, L., Corsini, E. M., Miralda-Escudé, J., Coccato, L., Sarzi, M., Pizzella, A., Beifiori, A. 2009, ApJ, 690, 537

Di Matteo, T., Springel, V., \& Hernquist, L. 2005, Nature, 433, 604

Emsellem, E., Monnet, G., \& Bacon, R. 1994, A\&A, 285, 723

Emsellem, E., et al. 2007, MNRAS, 379, 401

Emsellem, E., et al. 2007, MNRAS, 414, 888

Emsellem, E. 2013, MNRAS, 433, 1862

Faber. S. M. 1973, ApJ, 179, 423

Fabian, A. C. 1999, MNRAS, 308, L39

Fabian, A. C., Sanders, J. S., Haehnelt, M., Rees, M. J., \& Miller, J. M. 2013, MNRAS, 431, L38

Ferrarese, L., \& Merritt, D. 2000, ApJ, 539, L9

Gebhardt, K., et al. 2000, ApJ, 539, L13

Gebhardt, K., et al. 2003, ApJ, 583, 92

Gebhardt, K., \& Thomas, J. 2009, ApJ, 700, 1690

Gebhardt, K., Adams, J., Richstone, D., Lauer, T. R., Faber, S. M., Gültekin, K., Murphy, J., \& Tremaine, S. 2011, ApJ, 729, 119

Gonzaga, S., Hack, W., Fruchter, A, \& Mack, J., 2012, The DrizzlePac

Handbook (Baltimore, STScI)

Gültekin, K., et al. 2009, ApJ, 698, 198

Herriot, G., et al. 2000, Proc. SPIE, 4007, 115

Husemann, B., et al. 2013, A\&A, 549, 87

Jahnke, K., \& Macciò, A. V. 2011, ApJ, 734, 92

Jarrett, T. H., Chester, T., Cutri, R., Schneider, S., Skrutskie, M., \& Huchra, J. P. 2000, AJ, 119, 2498
Kelz, A., et al. 2006, PASP, 118, 129

Kewley, L. J., Groves, B., Kauffmann, G., \& Heckman, T. 2006, MNRAS, 372,961

Kormendy, J., \& Richstone, D. 1995, ARA\&A, 33, 581

Kormendy, J., et al. 1997, ApJ, 482, L139

Kormendy, J., \& Ho, L. C. 2013, ARA\&A, 51, 511

Krajnović, D., Cappellari, M., Emsellem, E., McDermid, R. M., \& de Zeeuw, P. T. 2005, MNRAS, 357, 1113

Krajnović, D., McDermid, R. M., Cappellari, M., \& Davies, R. L. 2009, MNRAS, 399, 1839

Krist, J., \& Hook, R. 2004, The Tiny Tim User's Guide (Baltimore:STScI)

Lauer, T. R., et al. 2007, ApJ, 662, 808

Marconi, A., \& Hunt, L. K. 2003, ApJ, 589, L21

McConnell, N. J., Ma, C.-P., Graham, J. R., Gebhardt, K., Lauer, T. R., Wright, S. A., \& Richstone, D. O. 2011, ApJ, 728, 100

McConnell, N. J., Ma, C.-P., Murphy, J. D., Gebhardt, K., Lauer, T. R.,

Graham, J. R., Wright, S. A., \& Richstone, D. O. 2012, ApJ, 756, 179

McConnell, N. J., \& Ma, C.-P. 2013, ApJ, 764, 184

McGregor, P. J., et al. 2003, Proc. SPIE, 4841, 1581

Monnet, G., Bacon, R., \& Emsellem, E. 1992, A\&A, 253, 366

Mould, J. R., et al. 2000, ApJ, 529, 786

Navarro, J. F., Frenk, C. S., \& White, S. D. M. 1996, ApJ, 462, 563

Onken, C. A., et al. 2014, ApJ accepted, arXiv:1406.6735

Peng, C. Y. 2007, ApJ, 671, 1098

Peng, C. Y., Ho, L. C., Impey, C. D., \& Rix, H.-W. 2010, AJ, 139, 2097

Roth, M. M., et al. 2005, PASP, 117, 620

Rusli, S. P., Thomas, J., Erwin, P., Saglia, R. P., Nowak, N., Bender, R. 2011, MNRAS, 410, 1223

Rusli, S. P., et al. 2013, AJ, 146, 45

Sánchez, S. F., et al. 2012, A\&A, 538, 8

Schlafly, E. F., \& Finkbeiner, D. P. 2011, ApJ, 737, 103

Schulze, A., \& Gebhardt, K. 2011, ApJ, 729, 21

Schwarzschild, M. 1979, ApJ, 232, 236

Seth, A. C., et al. 2010, ApJ, 714, 713

Seth, A. C., et al. 2014, Nature, 513, 398

Shen, J., \& Gebhardt, K. 2010, ApJ, 711, 484

Silk, J., \& Rees, M. J. 1998, A\&A, 331, L1

Thomas, J., Saglia, R. P., Bender, R., Thomas, D., Gebhardt, K., Magorrian,

J., Corsini, E. M., \& Wegner, G. 2005, MNRAS, 360, 1355

Toft, S., et al. 2014, ApJ, 782, 68 
Valdes, F., Gupta, R., Rose, J. A., Singh, H. P., \& Bell, D. J. 2004, ApJS, 152,251

van den Bosch, R. C. E., van de Ven, G., Verolme, E. K., Cappellari, M., \& de Zeeuw, P. T. 2008, MNRAS, 385, 647

van den Bosch, R. C. E., \& van de Ven, G. 2009, MNRAS, 398, 1117

van den Bosch, R. C. E., \& de Zeeuw, P. T. 2010, MNRAS, 401, 1770

van den Bosch, R. C. E., Gebhardt, Karl., Gültekin, K., van de Ven, G., van der Wel, A., \& Walsh, J. L. 2012, Nature, 491, 729

van den Bosch, R. C. E., Gebhardt, Karl., Gültekin, K., Yildirim, A., \& Walsh, J. L. 2014, ApJS, 218, 10

van der Marel, R. P., \& Franx, M. 1993, ApJ, 407, 525

van der Wel, A., et al. 2011, ApJ, 730, 38

van der Wel, A., et al. 2012, ApJS, 203, 24

van Dokkum, P. G., Kriek, M., \& Franx, M. 2009, Nature, 460, 717 van Dokkum, P. G., et al. 2010, ApJ, 709, 1018

Vazdekis, A., Casuso, E., Peletier, R. F., \& Beckman, J. E. 1996, ApJS, 106, 307

Verheijen, M. A., et al. 2004, Astron. Nachr., 325, 151

Walsh, J. L., Barth, A. J., \& Sarzi, M. 2010, ApJ, 721, 762

Walsh, J. L., van den Bosch, R. C. E., Barth, A. J., \& Sarzi, Marc. 2012, ApJ, 753, 79

Walsh, J. L., Barth, A. J., Ho, L. C., \& Sarzi, M. 2013, ApJ, 770, 86

Winge, C., Riffel, R. A., \& Storchi-Bergmann, T. 2009, ApJS, 185, 186

Yıldırım, A., van den Bosch, R. C. E., van de Ven, G., Husemann, B., Lyubenova, M., Walsh, J. L., Gebhardt, K., \& Gültekin, K. 2015 , submitted to MNRAS

Yıldırım, A., et al. 2014, in prep

Zirm, A. W., et al. 2007, ApJ, 656, 66 